\title{
J0454-0309: evidence of a strong lensing fossil group falling into a poor galaxy cluster ${ }^{\star}$
}

\author{
M. Schirmer ${ }^{1}$, S. Suyu ${ }^{1}$, T. Schrabback ${ }^{2}$, H. Hildebrandt ${ }^{2}$, T. Erben ${ }^{1}$, and A. Halkola ${ }^{3,4}$ \\ 1 Argelander-Institut für Astronomie, Universität Bonn, Auf dem Hügel 71, 53121 Bonn, Germany \\ e-mail: mischa@astro.uni-bonn.de \\ 2 Leiden Observatory, Leiden University, Niels Bohrweg 2, 2333 Leiden, The Netherlands \\ 3 University of Tuorla Observatory, Väisäläntie 20, 21500 Piikkiö, Finland \\ ${ }^{4}$ Excellence Cluster Universe, Technische Universität München, Boltzmannstr. 2, 85748 Garching, Germany
}

Received 5 December 2009 / Accepted 4 February 2010

\section{ABSTRACT}

\begin{abstract}
Aims. We have discovered a strong lensing fossil group (J0454) projected near the well-studied cluster MS0451-0305. Using the large amount of available archival data, we compare J0454 to normal groups and clusters. A highly asymmetric image configuration of the strong lens enables us to study the substructure of the system.

Methods. We used multicolour Subaru/Suprime-Cam and CFHT/Megaprime imaging, together with Keck spectroscopy to identify member galaxies. A VLT/FORS2 spectrum was taken to determine the redshifts of the brightest elliptical and the lensed arc. Using HST/ACS images, we determined the group's weak lensing signal and modelled the strong lens system. This is the first time that a fossil group is analysed with lensing methods. The X-ray luminosity and temperature were derived from XMM-Newton data.

Results. J0454 is located at $z=0.26$, with a gap of 2.5 mag between the brightest and second brightest galaxies within half the virial radius. Outside a radius of $1.5 \mathrm{Mpc}$, we find two filaments extending over $4 \mathrm{Mpc}$, and within we identify 31 members spectroscopically and 33 via the red sequence with $i<22$ mag. They segregate into spirals $\left(\sigma_{v}=590 \mathrm{~km} \mathrm{~s}^{-1}\right)$ and a central concentration of ellipticals $\left(\sigma_{v}=480 \mathrm{~km} \mathrm{~s}^{-1}\right)$, establishing a morphology-density relation. Weak lensing and cluster richness relations yield consistent values of $r_{200}=810-850 \mathrm{kpc}$ and $M_{200}=(0.75-0.90) \times 10^{14} M_{\odot}$. The brightest group galaxy (BGG) is inconsistent with the dynamic centre of J0454. It strongly lenses a galaxy at $z=2.1 \pm 0.3$, and we model the lens with a pseudo-isothermal elliptical mass distribution. A high external shear, and a discrepancy between the Einstein radius and the weak lensing velocity dispersion requires that the BGG must be offset from J0454's dark halo centre by at least $90-130 \mathrm{kpc}$. The X-ray halo is offset by $24 \pm 16 \mathrm{kpc}$ from the BGG, shows no signs of a cooling flow and can be fit by a single $\beta$-model. With $L_{\mathrm{X}}=(1.4 \pm 0.2) \times 10^{43} \mathrm{erg} \mathrm{s}^{-1} \mathrm{~J} 0454$ falls onto standard cluster scaling relations, but appears cooler $(T=1.1 \pm 0.1 \mathrm{keV})$ than expected $(T \sim 2.0 \mathrm{keV})$. Taken all together, these data indicate that J0454 consists of two systems, a sparse cluster and an infalling fossil group, where the latter seeds the brightest cluster galaxy. An alternative to the sparse cluster could be a filament projected along the line of sight mimicking a cluster, with galaxies streaming towards the fossil group.
\end{abstract}

Key words. galaxies: evolution - galaxies: clusters: individual: J0454-0309 - galaxies: formation

\section{Introduction}

* This work is based on data collected at the Subaru Telescope, which is operated by the National Astronomical Observatory of Japan; based on observations obtained with MegaPrime/MegaCam, a joint project of CFHT and CEA/DAPNIA, at the Canada-France-Hawaii Telescope (CFHT) which is operated by the National Research Council (NRC) of Canada, the Institut National des Sciences de l'Univers of the Centre National de la Recherche Scientifique (CNRS) of France, and the University of Hawaii; based on observations made with ESO Telescopes at the La Silla and Paranal Observatories, Chile (ESO DDT Programme 282.A-5066); based on observations made with the NASA/ESA Hubble Space Telescope (programme \#9836) obtained at the Space Telescope Science Institute, which is operated by the Association of Universities for Research in Astronomy, Inc., under NASA contract NAS 5-26555; based on observations obtained with XMM-Newton, an ESA science mission with instruments and contributions directly funded by ESA Member States and NASA; based on data obtained at the W.M. Keck Observatory, which is operated as a scientific partnership among the California Institute of Technology, the University of California and the National Aeronautics and Space Administration. The Observatory was made possible by the generous financial support of the W.M. Keck Foundation.
In a $\Lambda \mathrm{CDM}$ cosmology, galaxies acquire mass mostly through minor merger events, where one galaxy has 0.3 times or less the mass of its collision partner. Only the most luminous elliptical galaxies experience a major merger event in their history (Parry et al. 2009). The growth of large elliptical systems is facilitated particularly well in the low-velocity environments of galaxy groups where dynamical friction (Chandrasekhar 1943; Nusser \& Sheth 1999) is very efficient. This effect increases with the mass of the infalling galaxy and is higher for lower velocities. In this way galaxies cool down into the group or cluster core, losing their gas through ram-pressure stripping along the way (Quilis et al. 2000). At the same time they undergo slower morphological transformations (Parry et al. 2009; Scannapieco et al. 2009), leading to the formation of the red sequence in the inner region. The time scale for dynamical friction depends on the mass of the infalling galaxy and its distance from the core. For the most massive galaxies $\left(\sim 10^{12} M_{\odot}\right)$, it is as short as a few Gyr (Nath 2008; Boylan-Kolchin et al. 2008), 
implying that large elliptical galaxies in groups can already accur at early times.

Several mechanisms for the formation of BCGs (the brightest cluster galaxies) have been suggested, ranging from galactic cannibalism and cooling flows to merger processes during cluster collapse (see von der Linden et al. 2007, and references therein). Elliptical galaxies growing in this fashion should be located at the centre of the gravitational potential, and their recession velocity should match the mean of the radial velocities of the other cluster members for virialised systems. Recently, Skibba et al. (2010) have shown that in $\sim 40 \%$ of all haloes of mass $\sim 5 \times 10^{13} h_{100} M_{\odot}$ the BCG is not the central galaxy, falsifying this paradigm. This was also demonstrated for clusters with higher masses (Oegerle \& Hill 2001; von der Linden et al. 2007). Most of these analyses have in common that the centre of the halo is identified by the distribution centre of elliptical galaxies or, more rarely, by the X-ray centroid or weak gravitational lensing. Either of these methods has advantages and disadvantages; for instance, they can be hampered by small numbers of galaxies, low X-ray $S / N$ or projection effects. In this paper we are in the lucky situation that a strongly lensed galaxy let us put tight constraints on the dark matter halo centre, and in this way show that the BCG is not located at the minimum of the potential. We conclude that the BCG was formed outside the cluster in a nearby group, which is now falling into the cluster.

\subsection{Fossil groups}

Contrary to the quick dynamical collapse of galaxy groups, the cooling times for their X-ray haloes are comparable to one or several Hubble times (Sarazin 1988). This can lead to isolated giant elliptical galaxies, embedded in X-ray haloes with luminosities characteristic for entire galaxy groups. Such objects exist in the Universe (Vikhlinin et al. 1999), either isolated (Yoshioka et al. 2004) or surrounded by groups of less luminous satellite galaxies (Jones et al. 2003; Khosroshahi et al. 2006b; La Barbera et al. 2009). One of the first systems has been reported by Ponman et al. (1994) coining the term "fossil group", and Jones et al. (2003) have introduced general selection criteria. Accordingly, the galaxies must be embedded in an extended X-ray halo with $L_{X}>10^{42} h_{50}^{-2} \mathrm{erg} \mathrm{s}^{-1}$, integrated over the $0.5-2.0 \mathrm{keV}$ range. In addition, the central elliptical galaxy must be $\Delta m_{12}^{\min } \geq 2$ mag brighter in $R$-band than the second brightest galaxy (independent of morphology) within half the virial radius. This magnitude gap is motivated by the accretion of $L_{*}$-galaxies in the inner volume, which are then absent in the group's luminosity function. Current observational samples (e.g. Khosroshahi et al. 2007; La Barbera et al. 2009; Voevodkin et al. 2010) are largely based on this definition, and so are simulations (von Benda-Beckmann et al. 2008).

The selection criteria by Jones et al. (2003) have been relaxed in the course of systematic searches. Santos et al. (2007) have favoured a fixed radius of $0.5 h_{50} \mathrm{Mpc}$ within which the magnitude gap must hold, independent of the cluster's virial state. Voevodkin et al. (2010) adopt $0.7 r_{500} \sim 0.4 r_{\text {vir }}$, with $r_{500}$ being calculated from the group's X-ray luminosity. Similar relaxations have been adopted for the magnitude gap. Milosavljević et al. (2006) and La Barbera et al. (2009) show that there is no sharp transition in the magnitude gap of galaxy clusters, hence there is no physical motivation for a particular numeric value. Voevodkin et al. (2010) and La Barbera et al. (2009) favour smaller gap sizes of $\Delta m_{12}^{\min }=1.7$ and $1.75 \mathrm{mag}$, respectively. According to Voevodkin et al. (2010) the gap should not be too strict a requirement, as the determination of the total magnitude of very extended galaxies is not trivial.

As for the formation of the magnitude gap, von Benda-Beckmann et al. (2008) have found in simulations that it usually arises at redshifts $0<z<0.7$, after the haloes assembled half of their final mass at $0.8<z<1.2$. This is significantly earlier than the formation of normal groups (Dariush et al. 2007) and leads to increased NFW (Navarro et al. 1997) concentration parameters. Accordingly, the last major merger in the simulated fossil groups took place more than 6 Gyr ago for more than $50 \%$ of the galaxies. Most of the magnitude gaps are closed at later (current) times when more infall of satellite galaxies occurs.

The exact formation process of fossils is not yet entirely understood. For example, Yoshioka et al. (2004) observe mass-tolight ratios as high as 1000, which are difficult to explain if these galaxies assembled their mass only through dynamical friction. Another uncertainty lies in the type of the galaxies from which the giant elliptical forms. Khosroshahi et al. (2006b) argue that their disky isophotes indicate gas-rich mergers, which would distinguish these galaxies from the BCGs in normal clusters. These tend to show more boxy isophotes from gas-poor mergers. However, La Barbera et al. (2009) do not find a preference for either disky or boxy shapes in their larger sample. They have argued that fossils merely represent a transitional state in the last stages of mass assembly than a class of their own.

While the formation process of fossils is still a matter of debate, their occurrence is not. About 10-20\% of all X-ray luminous groups and clusters have fossil character (e.g. Jones et al. 2003; von Benda-Beckmann et al. 2008), with typical masses of $1-10 \times 10^{13} M_{\odot}$. However, they are difficult to identify observationally. Only a few dozen systems are known so far, mostly extracted from large-area surveys such as SDSS (Santos et al. 2007; La Barbera et al. 2009) or the 400D cluster catalogue (Voevodkin et al. 2010). The last authors discuss various difficulties in the selection process, in particular completeness and problems in the accurate determination of the magnitude of the brightest galaxy. In general, the observationally determined abundances of fossil groups agree with those predicted by simulations. However, in terms of absolute numbers samples are systematically incomplete since the second brightest galaxy can be at a sufficiently large physical distance from the centre and still appear projected onto the inner volume. Assuming that all galaxies are within the virial radius and follow a radially symmetric distribution, we estimate that $20 \%$ (25\%) of all fossils are overlooked for $r_{\min }=0.4(0.5) r_{\text {vir }}$ due to this effect.

Almost all of the few dozen fossil groups known were discovered and analysed based upon comparatively shallow optical and/or X-ray survey data. In general the observational data are poor compared to what are available for normal clusters. Only a few fossils were investigated in detail, such as ESO 3060170 (Sun et al. 2004), RX J1552.2+2013 (Mendes de Oliveira et al. 2006), RX J1416.4+2315 (Jones et al. 2003; Cypriano et al. 2006; Khosroshahi et al. 2006a), CL0259+0013 (Voevodkin et al. 2010) or UGC 842 (Lopes de Oliveira et al. 2010). For a comprehensive comparison with normal groups and clusters a systematic deep survey of a larger number is needed.

In this paper we present our analysis of J0454.0-0308 (hereafter: J0454), a fossil group at $z=0.26$. It is projected $8^{\prime}$ south of the well-known cluster MS0451-0301 (hereafter: MS0451, $z=0.54$ ), thus a large amount of archival data are available for our analysis. J0454 consists of at least 60 galaxies and was identified by us in Subaru/Suprime-Cam images. It is dominated by a giant elliptical galaxy (hereafter: E0454), which strongly lenses 


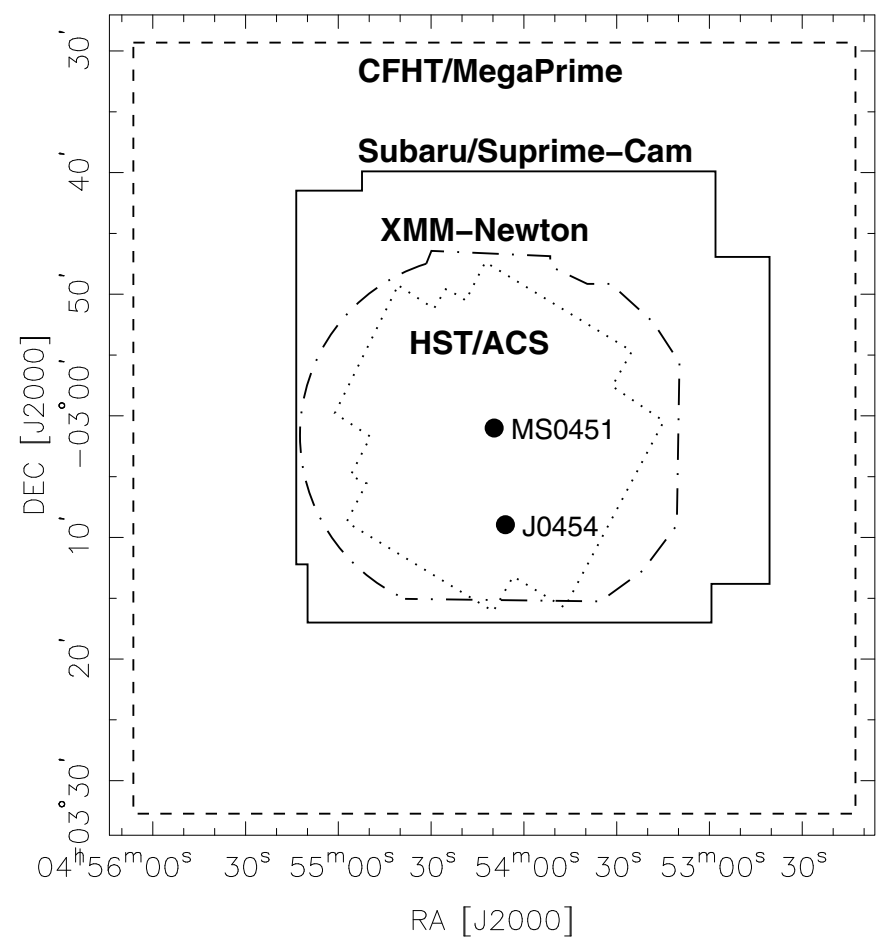

Fig. 1. Pointings of the imaging data sets. The positions of the fossil group J0454 and the background cluster MS0451 are shown as well.

a distant background source. We use Subaru/Suprime-Cam and CFHT/MegaPrime for photometry, XMM-Newton to study the intra-cluster gas and HST/ACS for the weak and strong lensing analysis. The imaging data (see Fig. 1 for an overview) are complemented by VLT and Keck spectroscopy.

\subsection{Terminology and assumptions}

In this work we present evidence that J0454 is composed of a poor cluster and an infalling fossil group. We refer to the global system as J0454, but also to the cluster without the fossil group. The latter distinction is only made in Sect. 8 when we discuss the results. E0454 is the brightest galaxy of the system.

The paper is organised as follows: in Sect. 2 we describe the imaging and spectroscopic data provided this was not done elsewhere. In Sect. 3 we study foreground and background contamination and select a red cluster sequence in colour-colour and colour-magnitude space. In Sect. 4 we investigate the galactic content of the system, establish a morphology-density relation and obtain the velocity dispersions of early- and late-type galaxies. We use virial properties and the size-richness relation for an estimate of $r_{200}$. In Sect. 5 we present the X-ray results, followed by our weak and strong lensing analysis in Sects. 6 and 7. We discuss our findings in Sect. 8 and summarise in Sect. 9.

We assume a flat standard cosmology with $\Omega_{\mathrm{m}}=0.27$, $\Omega_{\Lambda}=0.73$ and $H_{0}=70 \mathrm{hm} \mathrm{s}^{-1} \mathrm{Mpc}^{-1}$. On occasion we refer to relations from the literature with parameterisations $H_{0}=$ $100 h_{100} \mathrm{~km} \mathrm{~s}^{-1} \mathrm{Mpc}^{-1}$ or $H_{0}=50 h_{50} \mathrm{~km} \mathrm{~s}^{-1} \mathrm{Mpc}^{-1}$. To avoid confusion we quote them as published originally, indexing $h$ accordingly. X-ray luminosities are reported for the $0.5-2.0 \mathrm{keV}$ range, and optical luminosities are given in solar units. The relation between angular and physical scales at $z=0.26$ is $1^{\prime}=243 h^{-1} \mathrm{kpc}$. All numeric values quoted for physical distances in J0454 must be scaled with $h^{-1}$. Magnitudes are reported for both the Johnson-Cousins and the Sloan passbands and denoted with uppercase and lowercase letters, respectively. All error bars represent the $1 \sigma$ confidence level.

\section{Observations and data reduction}

\subsection{Subaru/Suprime-Cam and CFHT/Megaprime data reduction}

We serendipitously discovered J0454 in deep Subaru/SuprimeCam (Miyazaki et al. 2002) images of MS0451. The data were reduced with THELI $^{1}$ (Erben et al. 2005), our pipeline for the reduction of wide-field optical and near-infrared images. In the following we summarise those aspects where our reduction scheme deviated significantly from the standard approach.

Images were taken in nine different nights during six periods between 2001-01-22 and 2006-12-21 (PIs: H. Ebeling, N. Yasuda, G. Kosugi). Suprime-Cam consists of 10 CCDs, covering $34^{\prime} \times 27^{\prime}$ with $0^{\prime} .202$ per pixel. In 2001 Suprime-Cam had one broken CCD and individual gain settings. The defect CCD and three others were replaced, and the gains were homogenised and refined once more another year later. We brought all chips to the same gain and then performed the standard pre-processing including debiasing, flatfielding, superflatting, defringing, and sky subtraction. The data were astrometrically calibrated with Scamp (Bertin 2006) and then stacked. Since images were taken with two different sky position angles we could recover areas initially lost due to blooming. The data did not allow for correction of scattered light in the flat fields, for which extensive dithering of photometric standard fields is required (see Manfroid \& Selman 2001; Magnier \& Cuillandre 2004; Koch et al. 2004).

We complemented the BVRIz Subaru/Suprime-Cam data with $u^{*}$ griz CFHT/Megaprime images, which improves the photometric redshifts as a result of the presence of $u^{*}$-band. The CFHT/Megaprime data were pre-reduced using ELIXIR (Magnier \& Cuillandre 2004) at CFHT, including corrections for scattered light of the order of $0.1 \mathrm{mag}$. The remaining processing was done with THELI following Erben et al. (2009). The properties of the coadded images are summarised in Table 1.

\subsubsection{Catalogue creation}

Object detection and photometry was done using SExtractor (Bertin \& Arnouts 1996) in double image mode. We stacked all exposures in all filters of one camera with an image seeing of less than 1'.0, obtaining a deep noise-normalised detection image. Coadded images in the different filters were convolved to a common seeing of $0{ }^{\prime} 95$, ensuring that the object flux in each waveband was integrated over identical apertures. We kept objects with at least 5 connected pixels with $S / N \geq 2$ each.

The Subaru/Suprime-Cam data were only partially taken in photometric conditions, with zeropoint variations of up to $0.1 \mathrm{mag}$ in other nights. We tied the photometric $z$-band image to CFHT/Megaprime data taken in the same filter. The other Subaru/Suprime-Cam zeropoints were inferred by comparing the fluxes from non-saturated stars, measured in $3^{\prime \prime}$ wide apertures, against the Pickles (1998) library (for details see Erben et al. 2009). We took into account filter transmission, quantum efficiency, and the combined mirror reflectivity and corrector throughput (Table 2, S. Miyazaki, priv. comm.). The photometric calibration of the CFHT/Megaprime data was taken from the ELIXIR headers. The zeropoints of both data sets

\footnotetext{
1 Available at

http: //www . astro . uni-bonn . de/ mischa/theli .html
} 
Table 1. Summary of the Subaru/Suprime-Cam and CFHT/Megaprime data.

\begin{tabular}{|c|c|c|c|c|}
\hline Telescope/Instrument & Filter (abbr.) & $t_{\text {exp }}[\mathrm{s}]$ & Seeing & $M_{\text {lim }}$ \\
\hline Subaru/Suprime-Cam & WJB $(B)$ & 12240 & 0.82 & 26.7 \\
\hline Subaru/Suprime-Cam & WJV $(V)$ & 5040 & 0.95 & 26.0 \\
\hline Subaru/Suprime-Cam & $\operatorname{WCRC}(R)$ & 11400 & $0 .{ }^{\prime} 83$ & 26.6 \\
\hline Subaru/Suprime-Cam & $\operatorname{WCIC}(I)$ & 4920 & 0.92 & 25.9 \\
\hline Subaru/Suprime-Cam & WSZ $(z)$ & 4380 & 0.76 & 25.1 \\
\hline CFHT/Megaprime & $u^{*}$ & 5220 & 0.87 & 25.7 \\
\hline CFHT/Megaprime & $g$ & 3400 & $0 ! .85$ & 26.0 \\
\hline CFHT/Megaprime & $r$ & 14850 & 0.71 & 26.2 \\
\hline CFHT/Megaprime & $i$ & 1280 & 0.71 & 23.7 \\
\hline CFHT/Megaprime & $z$ & 1440 & $0 ! 70$ & 22.4 \\
\hline
\end{tabular}

Notes. The limiting AB magnitudes (50\% completeness limit) are for $10 \sigma$ point sources, and are on average 0.8 mag brighter for extended objects.

Table 2. Combined Subaru mirror reflectivity and corrector throughput.

\begin{tabular}{cc}
\hline \hline Wavelength $[\AA]$ & Throughput \\
\hline 4450 & 0.774 \\
5500 & 0.828 \\
6590 & 0.828 \\
7710 & 0.791 \\
9220 & 0.765 \\
\hline
\end{tabular}

were ultimately fine-tuned during the calculation of the photometric redshifts based on several hundred calibration spectra (see Sect. 2.1.2).

\subsubsection{Photometric redshifts}

We need photometric redshifts for the weak gravitational lensing analysis in Sect. 6, mainly to distinguish between lensed background and unlensed foreground galaxies. The photometric redshifts were obtained as outlined in Hildebrandt et al. (2009) for all objects in the catalogues (see Sect. 2.1.1) and calibrated against 774 and 493 spectroscopic redshifts from Moran et al. (2007), for CFHT and Subaru, respectively. Notice that Moran et al. (2007) obtained spectroscopic redshifts for a total of 1562 sources in the field of view of MS0451. We performed the phot- $z$ calibration using only those spectra of sources with photometric errors smaller than $0.1 \mathrm{mag}$ in all bands. In detail, we fix the redshifts of the corresponding galaxies to their spectroscopically determined values. The magnitude differences between the best-fit templates and the observed photometry then yield the zeropoint corrections, in the range of $0.02-0.09$ mag for CFHT and 0.04-0.18 mag for Subaru. The correlation between photometric and spectroscopic redshifts is shown in Fig. 2 for both data sets, using a confidence limit (ODDS parameter) higher than 0.8. Due to the lack of $u$-band data the Subaru photo-zs are highly unreliable for $z \lesssim 0.3$, moving a significant fraction of lensed galaxies into the unlensed foreground sample. The CFHT data are much better in this respect, but shows 2-3 times as much scatter for $z \gtrsim 0.6$ and fails for fainter galaxies due to inferior depth in $i$ and $z$-band. The accuracy of the photo-zs is $\sigma \sim 0.040$.

We run both data sets simultaneously through the photo- $z$ process but found the results to be significantly worse than the photo-zs obtained separately for CFHT and Subaru. This is due to different PSF characteristics of the two data sets which could not be homogenised sufficiently, and in particular due to the fact that the Subaru data could not be corrected for scattered light

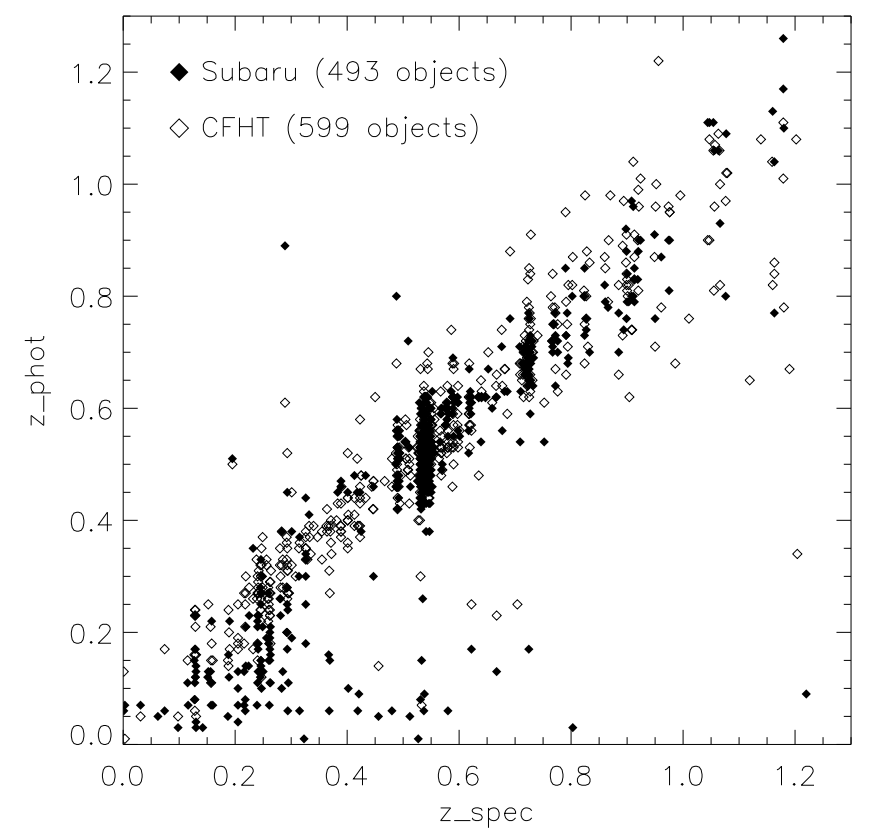

Fig. 2. Comparison of photometric and spectroscopic redshifts.

in the flats, resulting in inconsistent magnitudes across similar passbands. We therefore created a composite photo- $z$ catalogue in the following manner. We took the CFHT estimate if $z_{\text {phot }}^{\mathrm{CFHT}}<=0.4$ and the average if both estimates are between 0.4 and 0.7 . The remaining galaxies were split in two groups. The first is formed by galaxies for which the Subaru redshift is higher than 0.7 , and we assigned them this estimate. Galaxies in the second group, with $z_{\text {phot }}^{\text {Subaru }}<=0.7$ and $z_{\text {phot }}^{\text {CFHT }}>0.4$ got either the CFHT or the Subaru redshift assigned, depending on which one has higher confidence. Ultimately, the redshifts were transformed into relative lensing strengths,

$\beta=\frac{D\left(z_{l}, z_{s}\right)}{D\left(0, z_{s}\right)}$

where $D\left(z_{1}, z_{2}\right)$ is the angular diameter distance between two sources at redshifts $z_{1}$ and $z_{2}$, and $z_{l}$ and $z_{s}$ are the lens and source redshifts, respectively. See Sects. 2.2 and 6 for more details.

\subsection{HST/ACS imaging and shear catalogue}

For the weak lensing measurements and the strong lens modelling we rely on wide-field imaging with HST/ACS through the F814W filter (PI: R. Ellis). The data consist of 41 single orbit pointings of 2036s each, covering a continuous area of $19^{\prime} \times 19^{\prime}$, and was reduced according to Schrabback et al. (2007, 2009). An extensive description of our shape measurement pipeline is given in Schrabback et al. (2009). In the following we summarise the main characteristics.

The shear catalogue is based on SExtractor (Bertin \& Arnouts 1996) detections, for which we required a minimum number of 8 connected pixels with $S / N>1.4$ each after filtering with a $5 \times 5$ pixel wide Gaussian kernel. The object catalogue created in this manner was then fed into our implementation (see Erben et al. 2001) of the KSB method (Kaiser et al. 1995; Luppino \& Kaiser 1997; Hoekstra et al. 1998) for the shape measurement, adapted for HST/ACS as detailed in Schrabback et al. (2007, 2009). We employed a principal component interpolation for the variable HST/ACS point-spread function and parametric corrections for charge-transfer inefficiency for both stars and 
galaxies. In addition, we applied weights $w_{i}$ to the individual shear estimates given by

$w_{i}^{-1}=\left(\frac{2}{\operatorname{Tr}\left[P_{i}^{g}\right]}\right)^{2} \sigma_{e_{\mathrm{ani}}}^{2}\left(\operatorname{mag}_{i}\right)+0.25^{2}$,

where $\left(2 / \operatorname{Tr}\left[P_{i}^{g}\right]\right)$ is the isotropic PSF correction factor for galaxy $i$ and $\sigma_{e_{\text {ani }}}^{2}$ (mag) denotes the variance of the PSF anisotropy corrected galaxy polarisations fitted as a function of magnitude. We then selected galaxies with a minimum half light radius of $r_{\mathrm{h}}>1.2 r_{\mathrm{h}}^{* \text { max }}$, where $r_{\mathrm{h}}^{* \text { max }}$ is the maximum half light radius of the 0.25 pixel wide stellar locus in a size-magnitude diagram. An explicit magnitude cut was not performed. For more details we refer the reader to Schrabback et al. (2009).

After all filtering, the shear catalogue contains 33500 galaxies with redshift estimates $z>0.3$, corresponding to a number density of $n=73 \operatorname{arcmin}^{-2} .42 \%$ of the galaxies have their redshifts estimated photometrically as outlined in Sect. 2.1.2.

For those galaxies without redshift estimate (median magnitude $I_{F 814 W}=26.0$ ) we used the mean magnitude-redshift relation from Schrabback et al. (2009). Thereto we split the galaxies into magnitude bins of width $0.5 \mathrm{mag}$, starting from $I_{F 814 W}=23.0$ down to $I_{F 814 W}=27.5$. For each bin we calculated the average lensing strength $\langle\beta\rangle$ defined in Eq. (1). Since the lens is at a very low redshift of $z_{l}=0.26$, it is insensitive to the redshift distribution, in particular for galaxies with redshifts $z \gtrsim 0.7$. Essentially, $\langle\beta\rangle$ is between 0.70 and 0.80 for $96 \%$ of these galaxies, and we might as well have assumed a constant redshift without affecting our results.

The median and mean redshift of all galaxies in the shear catalogue are 1.39 and 1.16 , respectively. Objects are evenly distributed over the sky, and the area around J0454 has only very few masks for bright stars, none of which is larger than $\sim 20^{\prime \prime}$. We estimate the $50 \%$ completeness limiting $\mathrm{AB}$ magnitude of our shear catalogue to $I_{F 814 W} \sim 26.1 \mathrm{mag}$, consistent with the results for the COSMOS field, which was observed with very similar strategies (Scoville et al. 2007). The depth matches the one for the ground-based data (see Table 1).

\subsection{VLT/FORS2 spectroscopy of the strong lens system}

We used VLT/FORS2 to determine the redshifts of the fossil group's brightest elliptical, E0454, and its arc system. Data were taken on 2009-03-23 in DDT time and in 1".0 seeing, using the OG590 order sorting filter, GRIS_300I grism and a 1".0 long slit, resulting in a resolution of $R \sim 660\left(1.68 \AA\right.$ pixel $\left.^{-1}\right)$. The spectra were exposed for $2 \times 600 \mathrm{~s}$ and their useable range extends over 6250-9300 А. The long slit covered the core of E0454 and the bright northern arc.

We debiased, flat-fielded and sky-corrected the data using modified THELI modules. A third-order polynomial was fit to the calibration lamp emission lines for wavelength calibration, and a small residual offset was corrected by comparison to sky lines. We obtained the spectrum of E0454 by averaging 6 detector rows, yielding $S / N \sim 20$ in the continuum (Fig. 3). The spectrum of the arc is strongly blended with that of E0454. To remove this contamination, we exploited the symmetry of the lens and extracted a spectrum from the opposite side of E0454 at the same distance as the arc. This spectrum was subtracted from the arc's spectrum, which was then averaged over 4 rows yielding $S / N \sim 1-2$. The noise level was determined from 200 nearby detector rows which only contained sky background.

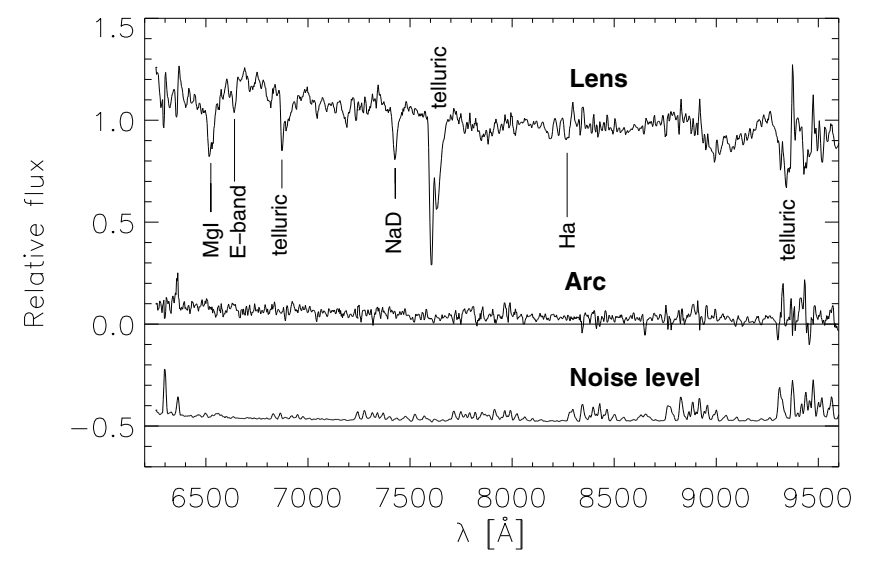

Fig. 3. Redshifted FORS2 spectra of the lens and the arc (binned 3 times). No significant features were found in the spectrum of the arc. The noise level was offset by -0.5 for better visibility.

The lens redshift is $z=0.2594 \pm 0.0004$ and based on five absorption features: $\mathrm{MgI} / \mathrm{MgH}(5156 / 5196 \AA)$, $E$-band (a blend of Fe and $\mathrm{Ca}$ at $5269 \AA)$, and $\mathrm{NaD}(5890 / 5896 \AA)$. Thus E0454 is a physical member of J0454, establishing the magnitude gap and thus the fossil character. The redshift of the arc is more difficult to infer. Our lens modelling (see Sect. 7) yields a magnification of 8-33 for the arc, which allows us to resolve two maxima in its light distribution. The colours of the object and the morphology rule out an early-type galaxy. If the morphology is indicative of star formation and if the redshift $\left(z_{\mathrm{arc}}\right)$ of the arc is less than about 1.0, then there would be a chance to detect the common set of nebular emission lines such as [OII] (3728 $\AA$ ), H $\beta$ (4863 $\AA$ ), [OIII] (5008 $\AA$ ) and $\mathrm{H} \alpha(6565 \AA)$ with the given exposure time. However, the spectrum does not contain any significant features. There are two possible explanations:

First, $z_{\text {arc }}$ is lower than $\sim 1.0$ and the morphology observed is not indicative for star formation, or the star formation rate (SFR) is low. In this case we can at least infer upper limits for the $S F R$ based on the non-detection of lines. For $z_{\text {arc }}=0.4$ the $\mathrm{H} \alpha$ line would still be accessible. Using Kennicutt (1998), a presumed line width of $30 \AA$ and correcting for the strong lens magnification (see Sect. 7), we find $S F R<0.15 M_{\odot} \mathrm{yr}^{-1}$. For $z_{\text {arc }}=0.7-1.0$ both [OII] and $\mathrm{H} \beta$ are covered. Following Argence \& Lamareille (2009) the upper limits for the SFR from these two lines are $S F R<0.2-2.5 M_{\odot} \mathrm{yr}^{-1}$ for $z_{\text {arc }}=0.7$ and $S F R<1-10 M_{\odot} \mathrm{yr}^{-1}$ for $z_{\mathrm{arc}}=1.0$, the uncertainties being due to the unknown $[\mathrm{OII}] / \mathrm{H} \beta$ line ratio.

The second possibility is that the lensed source is at significantly higher redshift, $z_{\text {arc }} \gtrsim 1.8$, such that possibly present emission lines are redshifted beyond the spectral range covered by our observations. The clear detection in $u^{*}$-band on the other hand means $z_{\text {arc }}<2.4$ and therefore $z_{\text {arc }}=2.1 \pm 0.3$. Based on strong-lensing properties and the stellar velocity dispersion of E0454 we show in Sect. 7.2 that this higher redshift is indeed the most plausible assumption. The actual redshift of the arc is not relevant for our main conclusions (see Sect. 8.1).

\subsection{X-ray observations}

The field was observed for $42 \mathrm{ks}$ on 2004-09-17 with XMM-Newton (PI: D. Warroll, observation ID 0205670101), covering a radius of $\sim 14$ around MS0451. J0454 is contained in the 2XMM catalogue (Watson et al. 2009) as source 2XMM J045400.6-030832:41489. We reduced the data 

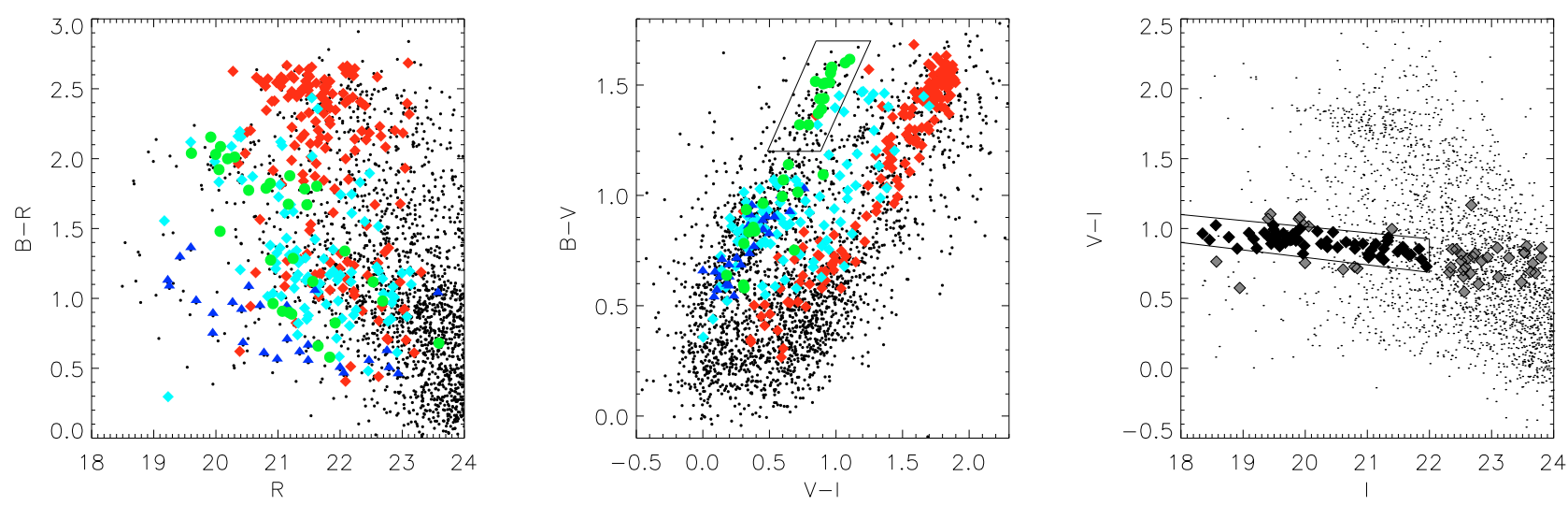

Fig. 4. Target selection in colour-colour and colour-magnitude space. The left and middle panels: galaxies with $0.52<z<0.56$ and $0.28<z<0.5$ are shown as red and cyan diamonds, respectively. Confirmed members of J0454 are coded green, and blue triangles are objects with $0.1<z<0.24$. A selection in colour-magnitude space leads to significant contamination with objects at higher redshifts (left panel, exemplary for $B-R$ vs. $R$ ). Instead, we selected galaxies in $B-V$ vs. $V-I$ (middle). The right panel shows that the galaxies selected in $B-V$ vs. $V-I$ form a well-defined red sequence in $V-I$ vs. $I$, and the box indicates additional selection criteria. Black points represent galaxies that were kept based on this purely photometric selection, and grey ones were excluded. Small corrections were made by means of available spectra (see text for details).

using XMM-SAS ${ }^{2}$ v8.0.0. The maximum flare level in the $10-15 \mathrm{keV}$ range is well below 0.35 counts s$^{-1}$, and about half of the data were taken during completely quiescent periods. Thus we did not reject any data due to high background rates.

$\mathrm{X}$-rays are particularly absorbed by neutral hydrogen,

$I(E)=I_{0} \mathrm{e}^{-\sigma_{\mathrm{ph}}(E) N_{\mathrm{HI}}}$

where $N_{\mathrm{HI}}=3.53 \times 10^{20} \mathrm{~cm}^{-2}$ is the column density along the line of sight (taken from Kalberla et al. 2005). Assuming that all hydrogen atoms are in their ground state, we obtained the quantum mechanical photon cross section as

$\sigma_{\mathrm{ph}}(E)=1.61 \times 10^{-23} \mathrm{~cm}^{2}\left(\frac{E}{\mathrm{keV}}\right)^{-3.5}$.

Accordingly, the absorption is significant for low X-ray energies $(0.2-0.5 \mathrm{keV})$ and becomes low for energies higher than $0.5-1 \mathrm{keV}$ (see also Morrison \& McCammon 1983). For the soft cluster spectrum of J0454 ( $T=1.1 \mathrm{keV}$, see Sect. 5), $2.5 \%$ of the flux are absorbed in the $0.5-2.0 \mathrm{keV}$ range. We also applied a $k$-correction factor of 1.06 , interpolated from the values tabulated by Böhringer et al. (2004).

\section{Cluster members and field contamination}

\subsection{Object selection}

The MS0451 field had extensive wide-field spectroscopy with Keck by Moran et al. (2007), who kindly made their redshift catalogue publicly available. They randomly selected galaxies in a Subaru I-band image (a subset of the data we use) from a sample with $I<21.5 \mathrm{mag}$, irrespective of morphology. Remaining spaces in the 14 slit masks were then filled up with fainter objects. In total, redshifts were obtained for 1562 galaxies in a $25^{\prime} \times 20^{\prime}$ field that covers J0454 as well.

\subsubsection{Selection of J0454 member galaxies}

The spectroscopic sampling of J0454 is complete to about $44 \%$ for $I<21.5$ mag (see Sect. 3.1.3). First, J0454 is at significantly lower redshift than MS0451, and thus its brighter galaxies were not observed as it is implausible that they are members

2 http://xmm.esac.esa.int/sas/ of MS0451. This holds in particular for the central elliptical with $I=16.6$ mag. Second, its angular separation from MS0451 is $8^{\prime}$ and thus it was not at the centre of interest. Lastly, slit masks cannot be configured arbitrarily due to source clustering.

Our photometric redshifts $\left(\sigma_{\text {photz }}=0.040\right)$ do not offer sufficient power to distinguish unambiguously between structures in the range $z=0.24-0.32$, which are present along the line of sight (Sect. 3.1.3). For a more complete picture we therefore selected ellipticals in colour-colour and colour-magnitude space using the red cluster sequence (RCS) method from Gladders \& Yee (2000). The spectroscopic redshifts were used to identify suitable areas. However, the large angular extent of MS0451 and its significant content of blue galaxies leads to a high contamination when using the red sequence alone (see e.g. Fig. 4, left panel). We investigated various colour-colour combinations and found that in $B-V$ vs. $V-I$ (Fig. 4, middle panel) the highest redshift differentiation is achieved. All galaxies at $z \sim 0.26$ are cleanly separated from those at $z=0.54$, thus removing the bulk of the contamination. There is also very little overlap with galaxies at $z \geq 0.3$. Only bluer objects at $z=0.26$ cannot be separated from those at lower redshift. In a first pass, we selected objects with

$$
\begin{aligned}
& B-V>1.2 \\
& B-V<1.7 \\
& B-V>1.286(V-I)+0.07 \\
& B-V<1.286(V-I)+0.59
\end{aligned}
$$

These form a red sequence in $V-I$ vs. $I$ (diamonds in the right panel of Fig. 4) with a typical width of $\sigma=0.049$ (see e.g. Hansen et al. 2009). Only objects within $2 \sigma$ of the red sequence and with $I \leq 22$ are kept for later analysis. The $I<22$ cut-off was chosen for two reasons. First, the width of the red sequence increases significantly for fainter galaxies (see right panel of Fig. 4), and thus the contamination rate would increase as well. Second, the Keck spectroscopic survey is limited by $I \lesssim 21.5$. Pushing the photometrically selected sample significantly beyond this limit would mean that we could not quantify anymore the contamination rate by structures with similar redshifts.

Taken all together, these selection criteria exclude all galaxies with $z_{\text {spec }}<0.24$, and all but two ellipticals with $z_{\text {spec }}>0.29$. 


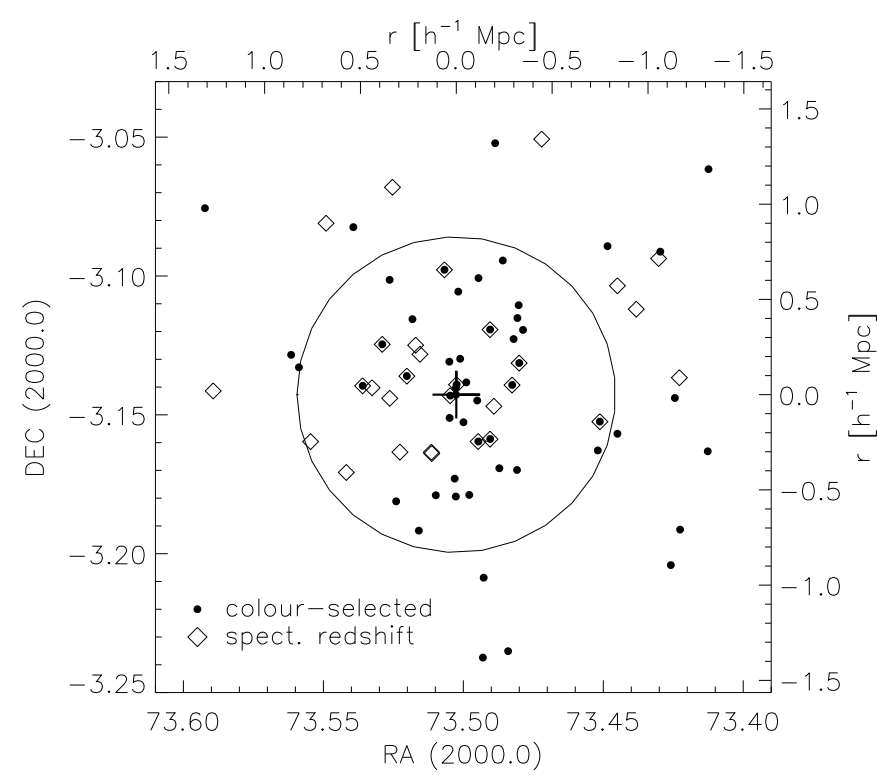

Fig. 5. Photometrically and spectroscopically selected cluster galaxies. The circle indicates $\left\langle r_{200}\right\rangle=830 \mathrm{kpc}$, centred on E0454.

A good fit to the red sequence formed by the remaining galaxies is

$V-I=-0.0430 I+1.768$.

From the sample of 55 galaxies selected in this manner (black diamonds in the right panel of Fig. 4) we removed three with higher and one with lower spectroscopic redshift, and those where the photometric redshifts deviated by more than 0.1 from the cluster redshift ( $2.5 \sigma$ rejection, 4 objects). In total, 47 galaxies remained to which we added 17 with confirmed redshifts, most of them galaxies with blue colours. One galaxy (object \#10) had colours redder than the red sequence (caused by a prominent dust lane) and was added back to the sample.

In total, 15 of the red sequence galaxies have spectroscopic redshifts, including the red galaxy that was added back to the sample. Assuming that similar effects hold for the 32 red sequence galaxies without spectra, we estimate that about 2 galaxies were overlooked. Our sample of red sequence galaxies is then $95 \%$ complete down to $i=22.0 \mathrm{mag}\left(M_{i}=-18.6 \pm 0.05\right)$.

We confined the galaxy sample to within $6^{\prime}$ of the brightest elliptical galaxy, E0454, corresponding to $1.7\left\langle r_{200}\right\rangle$ (see Sects. 4.3 and 6.2). Beyond this perimeter the number density of red sequence galaxies is indistinguishable from the density of field galaxies selected in the same manner $(n=$ $0.09 \mathrm{arcmin}^{-2}$, determined from a $10^{\prime} \times 11^{\prime}$ wide area where structures with $0.2<z<0.3$ are unknown).

After correcting for galactic extinction (Schlegel et al. 1998) we determined the $k$-correction (Hogg et al. 2002) using kcorrect (v. 4.1.4, Blanton \& Roweis 2007). For better comparison with other publications we report the rest-frame absolute magnitudes in the Sloan $g$ and $i$ passbands. The errors for $M_{g}$ and $M_{i}$ are 0.07 and $0.05 \mathrm{mag}$, respectively, based upon measurement uncertainties and the internal error estimate of kcorrect. The spatial distribution of the member galaxies is shown in Fig. 5, and their properties are summarised in Table A.1.

\subsubsection{Magnitude gap}

Assuming a virial radius of $\left\langle r_{200}\right\rangle=840 \mathrm{kpc}$ (3.5) from our analysis presented below, we determined a magnitude gap of
$\Delta m_{12}=2.5$ mag in $I$-band for J0454 within half the virial radius. The second-brightest galaxy is object \#20 from Table A.1, an elliptical galaxy at a separation of $0.46\left\langle r_{200}\right\rangle$ and with spectroscopic confirmation of its redshift. Notice that within $0.5\left\langle r_{200}\right\rangle$ there is no other possible foreground or background galaxy brighter than the second-brightest member galaxy, hence the fossil character of J0454 is secured. The third- and fourth-brightest members within $0.5\left\langle r_{200}\right\rangle$ are 2.8 mag fainter than E0454 and also spectroscopically confirmed. Two brighter galaxies exist at larger radii with $\Delta_{m}=1.8-1.9$ (objects \#34 and \#45), but they do not have their redshifts measured. For a meaningful luminosity function we need complete spectroscopic sampling, in particular because the line of sight is contaminated by nearby structures in redshift space (see Sect. 3.1.3).

\subsubsection{Structures along the line of sight}

Based on the Keck spectra we identified 16 structures between $0.1<z<0.8$, consisting of at least 12 galaxies within $\Delta z=0.01$. The spatial distributions of the 12 most significant ones are shown in Fig. 6. The circle indicates $\left\langle r_{200}\right\rangle=830 \mathrm{kpc}$ determined below from galaxy counts (Sect. 4.3) and weak gravitational lensing (Sect. 6.2). MS0451 overlaps significantly with J0454, whereas other structures contribute fewer interlopers.

The distributions shown in Fig. 6 are representative of the actual galaxy distribution. This is not self-evident due to the incomplete spectroscopic sampling with slit masks. However, the main selection criterion of Moran et al. (2007) was simply $I<21.5 \mathrm{mag}$, with a possible bias preferring galaxies closer to MS0451 over those with larger separations. Thus the selection function is approximately constant across the field and does not favour one particular structure over another.

The line of sight towards J0454 is not only contaminated by MS0451 but also by structures at $z=0.240,0.246,0.282,0.293$ and 0.325 . Without spectra we cannot distinguish these from members at $z=0.26$. We estimated the contamination assuming that the interlopers had the same probability of being selected for spectroscopy as the members of J0454. From the number of red sequence galaxies with and without spectra we determined the spectroscopic coverage to be $44 \%$ complete for $I<21.5$. Five interlopers were kept by the initial selection (see Sect. 3.1.1) and therefore we expect that about 10 of the 32 purely photometrically selected galaxies in Table A.1 are not true members of J0454. We applied corrections for this where necessary.

\section{Morphology-density relation, kinematics and $\boldsymbol{r}_{200}$}

In this section we show that J0454 has characteristics typical for normal galaxy clusters, such as a distinct morphology-density relation (see e.g. Goto et al. 2003) and a significantly lower velocity dispersion for the central population of elliptical galaxies as compared to the population of spirals. Based upon general cluster scaling relations, we obtain size and mass estimates.

\subsection{Cluster extent and mass: $r_{200}$ and $M_{200}$}

A characteristic key estimate of a cluster's linear extent is the virial radius. It is often approximated by $r_{200}$, within which the mean density is 200 times higher than the critical density $\rho_{\mathrm{c}}$,

$\rho_{\mathrm{c}}(z)=\frac{3}{8 \pi G} H^{2}(z)$, with

$H^{2}(z)=H_{0}^{2}\left[\Omega_{\mathrm{m}}(1+z)^{3}+\Omega_{\Lambda}\right]$ 


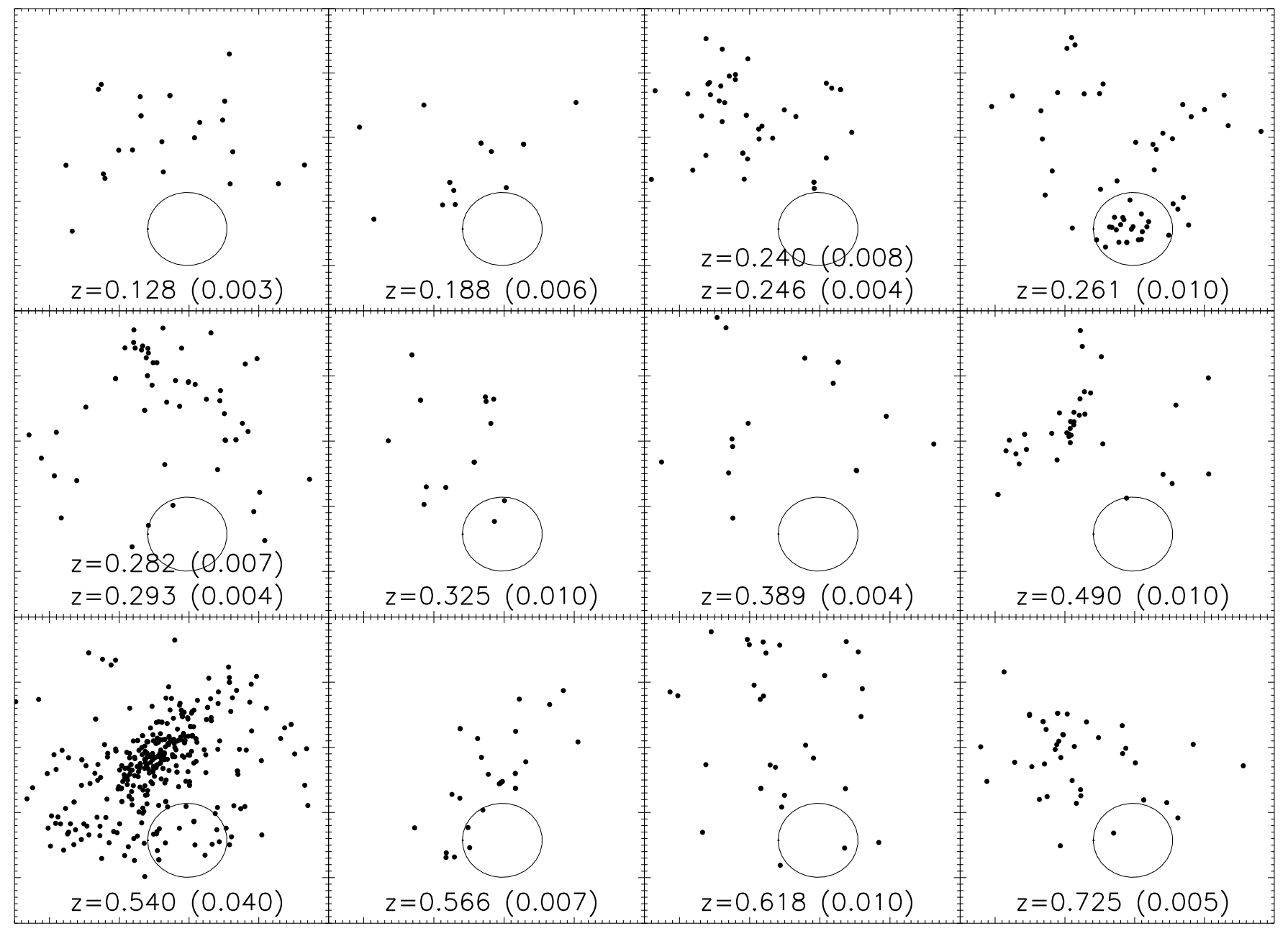

Fig. 6. Clustering for different spectroscopic redshift bins and their width (in parentheses). J0454 is shown in the upper right, MS0451 in the lower left. The circle is centred on E0454 and traces $\left\langle r_{200}\right\rangle=830 \mathrm{kpc}$ at $z=0.26$. North is up and East is left. The field is $25^{\prime}$ wide and centred on $\alpha=$ 04:54:06, $\delta=-03: 02: 06$.

being the Hubble function. The mass enclosed within $r_{200}$ is

$M_{200}=200 \rho_{\mathrm{c}}(z) \frac{4 \pi}{3} r_{200}^{3}$

A common estimator for the virial mass is

$M_{200}^{\text {dyn }} \sim \frac{3 \sigma_{v}^{2}}{G} r_{200}$

which can be combined with (10) and (12) yielding a dynamic estimate for $r_{200}$,

$r_{200}^{\mathrm{dyn}}=\frac{\sqrt{3}}{10} \frac{\sigma_{v}}{H(z)}$.

Estimating virial masses from galaxy dynamics is non-trivial (see e.g. Carlberg et al. 1997), in particular if the cluster under investigation is poorly sampled with spectroscopic redshifts. Our dynamic mass and size estimates for J0454 should therefore be viewed with caution, and we complement them with more robust cluster scaling relations, weak lensing and X-ray estimates.

\subsection{Velocity field and virial estimate of $r_{200}$}

In Fig. 7 we show the positions of all galaxies around J0454 with spectroscopic redshifts in the range $0.255<z<0.265$.

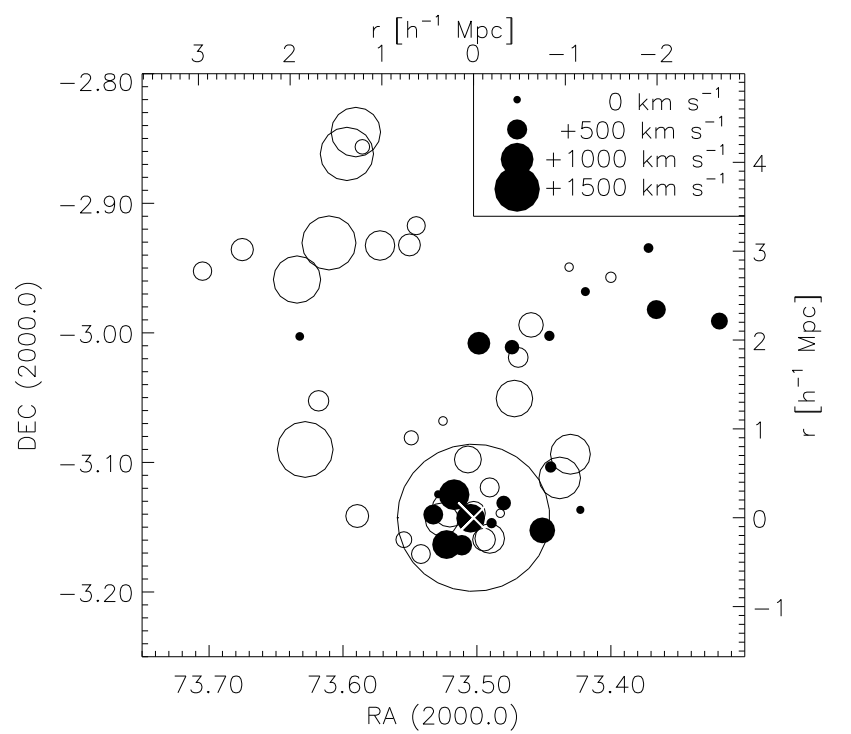

Fig. 7. The kinematic structure of J0454 with respect to E0454. Filled and open symbols mark red- and blueshifted galaxies, respectively.

The symbol size encodes the relative velocity with respect to E0454, and open (filled) symbols denote blueshifted (redshifted) 
motions. We notice two filaments extending up to $4.3 \mathrm{Mpc}$ to the North and to the North-West. The former is on average blueshifted by $-595 \mathrm{~km} \mathrm{~s}^{-1}$ compared to E0454, whereas the latter does not show a significant motion. A photometric selection of more member galaxies in these areas would result in significant contamination as these filaments are projected onto four structures at similar redshifts (Fig. 6). We thus confined our subsequent analysis to the region within $6^{\prime}$ from E0454.

We compute the velocity dispersion $\sigma_{v}$ as

$\sigma_{v}^{2}=\left(\frac{c}{1+\langle z\rangle}\right)^{2}\left(\frac{1}{N-1} \sum_{i}\left[z_{i}-\langle z\rangle\right]^{2}-\langle\delta\rangle^{2}\right)$,

excluding E0454 and following the prescription of Danese et al. (1980) and Harrison (1974). Therein, $c$ is the speed of light, $\langle z\rangle$ the mean cluster redshift, and $\langle\delta\rangle$ the uncertainty in the redshift measurement $\left(50 \mathrm{~km} \mathrm{~s}^{-1}\right.$, from Moran et al. 2007). The factor $(1+\langle z\rangle)^{-1}$ cancels the stretching effect of cosmic expansion. After the visual classification of the galaxies' morphologies based on their appearance in the HST/ACS data, we determined $\sigma_{v}$ for the red (E, S0) and the blue (Sa-Sc, Irr) population and for all galaxies together (see left panel of Fig. 8). Including a correction for local peculiar motions (Rakić et al. 2006) we have $\sigma_{v}^{\text {red }}=480 \pm 20 \mathrm{~km} \mathrm{~s}^{-1}, \sigma_{v}^{\text {blue }}=590 \pm 20 \mathrm{~km} \mathrm{~s}^{-1}$, and $\sigma_{v}^{\text {all }}=570 \pm 20 \mathrm{~km} \mathrm{~s}^{-1}$. The errors were obtained from the propagated mean measurement error, and include a conservative estimate for the uncertainty of the local peculiar motion and a possible net motion of J0454.

The velocity dispersion of the red galaxies is significantly lower than the one of the blue galaxies, which is expected from dynamical friction and the morphology-density relation (right panel of Fig. 8, consistent with the findings of Goto et al. 2003, for a much larger sample of clusters). Their mean velocities are different too, and offsets exist with respect to E0454 $\left(+240 \mathrm{~km} \mathrm{~s}^{-1}\right.$ for the red population, significant on the $2.5 \sigma$ level, and $+540 \mathrm{~km} \mathrm{~s}^{-1}(5.7 \sigma)$ for the blue galaxies). For the red galaxies this could still be an observational effect due to incomplete sampling, as within $1^{\prime}$ of E0454 only two of nine ellipticals have their redshifts measured. If confirmed by future observations, these features would indicate that these galaxies have a different origin than those which already collapsed into E0454, and that significant substructure exists in the entire system (see also Oegerle \& Hill 2001).

Using Eqs. (13) and (14) we obtained $r_{200}^{\text {dyn }}=1054 \pm 44 \mathrm{kpc}$ and $M_{200}^{\text {dyn }}=(1.69 \pm 0.14) \times 10^{14} M_{\odot}$ for the red population, and $r_{200}^{\text {dyn }}=1295 \pm 44 \mathrm{kpc}$ and $M_{200}^{\text {dyn }}=(3.14 \pm 0.21) \times 10^{14} M_{\odot}$ for the blue population.

\subsection{Size-richness relation}

Hansen et al. (2005) and Johnston et al. (2007) have shown that $r_{200}$ and $M_{200}$ can be estimated starting from the number $N_{\text {gal }}$ of galaxies within a radius of $1 h_{100}^{-1} \mathrm{Mpc}$ of the BCG. Only galaxies in the red sequence and with $i$-band luminosities $L>0.4 L_{*}$ are considered. Based on $N_{\text {gal }}$ one has

$r_{200}^{\mathrm{gal}}=0.156 h_{100}^{-1} \mathrm{Mpc} N_{\mathrm{gal}}^{0.60}$,

a refined version of the original relation from Hansen et al. (2005). Within $r_{200}^{\text {gal }}$ the luminosity is 200 times the mean luminosity of the Universe. It must not be mistaken for $r_{200}$ which refers to matter overdensity, yet the two are closely related (Johnston et al. 2007). Based on weak lensing measurements and
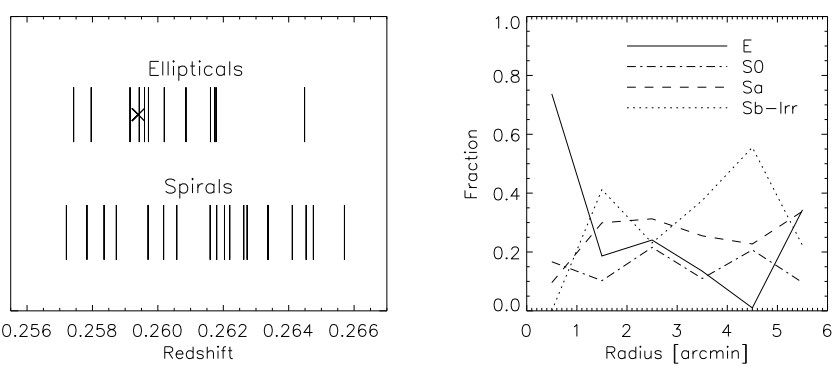

Fig. 8. Left panel: redshift distribution for the red (E, S0) and blue (Sa-Sc, Irr) galaxy populations. Notice that the spectroscopic sampling of ellipticals is complete to only $\sim 44 \%$. The cross marks E0454. Right panel: Galaxy types as a function of angular separation from E0454.

the number $N_{200}$ of galaxies within $r_{200}^{\text {gal }}$, Johnston et al. (2007) and Hansen et al. (2009) obtain

$r_{200}=0.182 h_{100}^{-1} \operatorname{Mpc} N_{200}^{0.42}$

$M_{200}=1.75 \times 10^{12} h_{100}^{-1} M_{\odot} N_{200}^{1.25}$

Using $M_{i, *}=-21.8$ mag from Hansen et al. (2009), we counted $N_{200}=15_{-2}^{+1}$ red sequence galaxies with $M_{i}<-20.8$ mag (corresponding to $0.4 L_{*}$ ). This richness estimate contains a correction for field contamination, and the errors are due to an uncertainty of 0.2 mag which we allowed for $M_{i, *}$. As a result we have $r_{200}=811 \pm 46 \mathrm{kpc}$ and $M_{200}=(0.74 \pm 0.15) \times 10^{14} M_{\odot}$, including $13 \%$ intrinsic uncertainty for the mass-richness relation.

\section{X-ray halo}

The XMM-Newton image of J0454 is shown in Fig. 9, overlaid over the HST/ACS optical image, and in Fig. A.1 in the Appendix (overlaid over a colour picture of the Subaru/SuprimeCam data, online material). X-ray flux is detected locally out to $1^{\prime}(240 \mathrm{kpc})$ from the core of E0454, encompassing the $10 \mathrm{in}-$ nermost galaxies. If azimuthally averaged, we can trace the halo about twice as far. It is possible that this very extended emission is not associated with E0454 anymore but with the surrounding cluster of galaxies (see below). The offset of the X-ray centroid with respect to E0454 is $6^{\prime \prime} \pm 4^{\prime \prime}(24 \mathrm{kpc})$. The luminosity profile is described by an isothermal $\beta$-model with $\beta=0.57 \pm 0.06$ and a core radius of $r_{\mathrm{c}}=120 \pm 17 \mathrm{kpc}$ (Fig. 10). The bestfit isothermal redshifted bremsstrahlung model of the spectrum yields $T=1.1 \pm 0.1 \mathrm{keV}$. Assuming a mean particle mass of $\mu=0.6$ we find $M_{200}=(0.34 \pm 0.10) \times 10^{14} M_{\odot}$ and $r_{200}=617 \pm$ $28 \mathrm{kpc}$, respectively, and for the total luminosity within $r_{200}$ we have $L_{\mathrm{X}}=(1.4 \pm 0.2) \times 10^{43} h^{-2} \mathrm{erg} \mathrm{s}^{-1}$.

A cooling flow is absent from the data as can be seen from the luminosity profile. Consequently, we do not expect star formation in the core of E0454. This is confirmed by our VLT/FORS2 spectrum (Fig. 3) which does not show any $\mathrm{H} \alpha$-emission, which would be a prime indicator for star formation second to molecular CO emission (Edge 2001).

The X-ray properties of J0454 agree with those of normal groups and clusters. Rykoff et al. (2008) find a tight correlation between $\left\langle L_{X}\right\rangle$ and $\left\langle N_{200}\right\rangle$ of 17000 maxBCG clusters, and this relation describes J0454 well. The $L_{\mathrm{X}}-\sigma$ relation drawn from the same sample predicts $\sigma \sim 480 \pm 30 \mathrm{~km} \mathrm{~s}^{-1}$, the same as we measured for the elliptical galaxy population. In the compilation of Mulchaey (2000) J0454 falls comfortably within the natural 


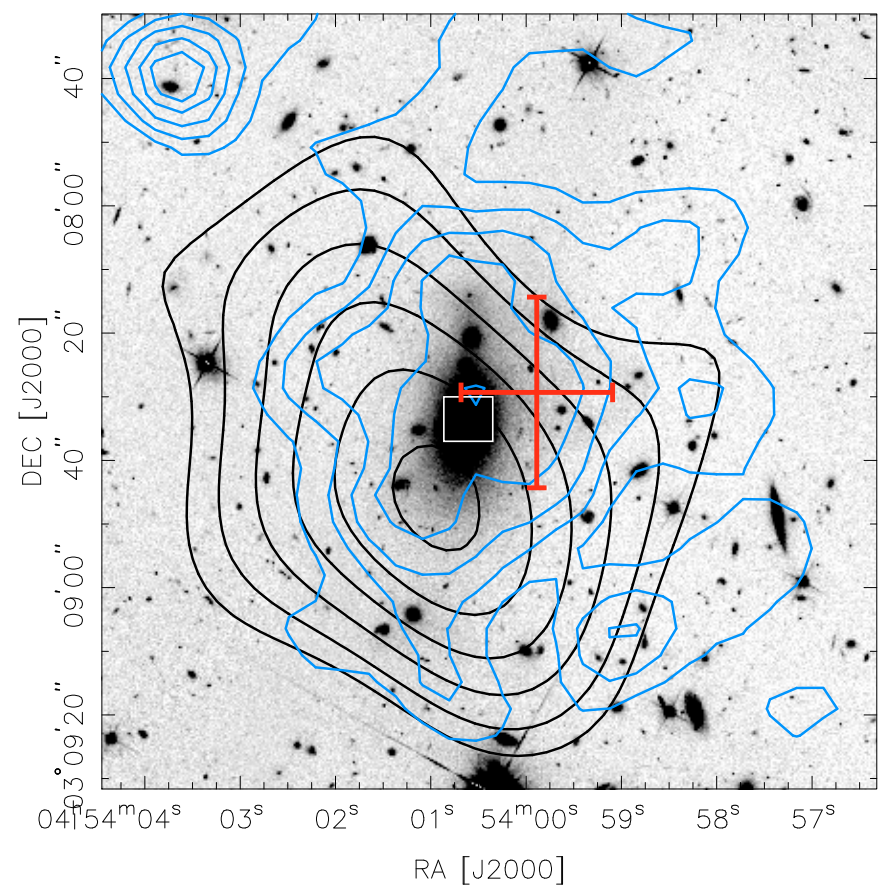

Fig. 9. HST/ACS image of J0454. The (jagged) blue contours trace the $S / N$-ratio of the $0.5-2.0 \mathrm{keV} \mathrm{X}$-ray flux, starting with $3 \sigma$ and increasing in steps of $2 \sigma$. A $6^{\prime \prime}$ wide kernel was used for smoothing. The (smooth) black contours trace the $S / N$ of the weak lensing mass reconstruction, starting with $2 \sigma$ and increasing in steps of $0.5 \sigma$, smoothed with a $40^{\prime \prime}$ wide kernel. The white square outlines the area of the strong lensing system shown in Fig. 12, and the red cross marks the centroid of the distribution of elliptical galaxies within $\left\langle r_{200}\right\rangle \sim 830 \mathrm{kpc}$.

scatter of the $L_{\mathrm{X}}-\sigma$ relation, resembling either a rich group or a poor cluster.

Differences occur in temperature-based scaling relations. While no deviation is found with respect to the $L_{\mathrm{X}}-T$ relation from the HIFLUGCS sample (Stanek et al. 2006; Reiprich \& Böhringer 2002), J0454 appears cooler than expected ( 2 keV) when comparing it to the $L_{X}-T$ relations presented by Mulchaey (2000) and Rykoff et al. (2008). A similar trend is seen for $T-\sigma$ (Mulchaey 2000), i.e. for $\sigma=480 \mathrm{~km} \mathrm{~s}^{-1}$ one would expect $T \sim 2.0 \mathrm{keV}$ (or $\sigma \sim 330 \mathrm{~km} \mathrm{~s}^{-1}$ for $T=1.1 \mathrm{keV}$ ). These deviations can be explained by the natural scatter seen in groups of galaxies. A different explanation would be that we see a groupsized substructure embedded in, but not yet fully merged with, a larger sparse cluster. Extended and patchy X-ray emission exists on the lowest levels and at radii $\gtrsim 1^{\prime}$. It is unclear whether this emission is still part of the E0454 halo or if we see the brightest emission features of the gas associated with J0454. With deeper $\mathrm{X}$-ray data we could look for temperature variations or different chemical compositions to distinguish these two components. We discuss these findings in Sect. 8.

The inner, flat core of the X-ray halo is elongated, tracing the optical ellipticity of E0454. These trends have been seen previously for groups (e.g. Mulchaey \& Zabludoff 1998) and clusters (e.g. Hashimoto et al. 2008), and also for fossils (Khosroshahi et al. 2004; Sun et al. 2004; Khosroshahi et al. 2006a). In general, the X-ray contours of the halo analysed in this work are not as concentric and regular as e.g. those for the fossil groups RX J1331.5+1108 and RX J1416.4+2315 from Khosroshahi et al. (2007), yet they do not appear more disturbed

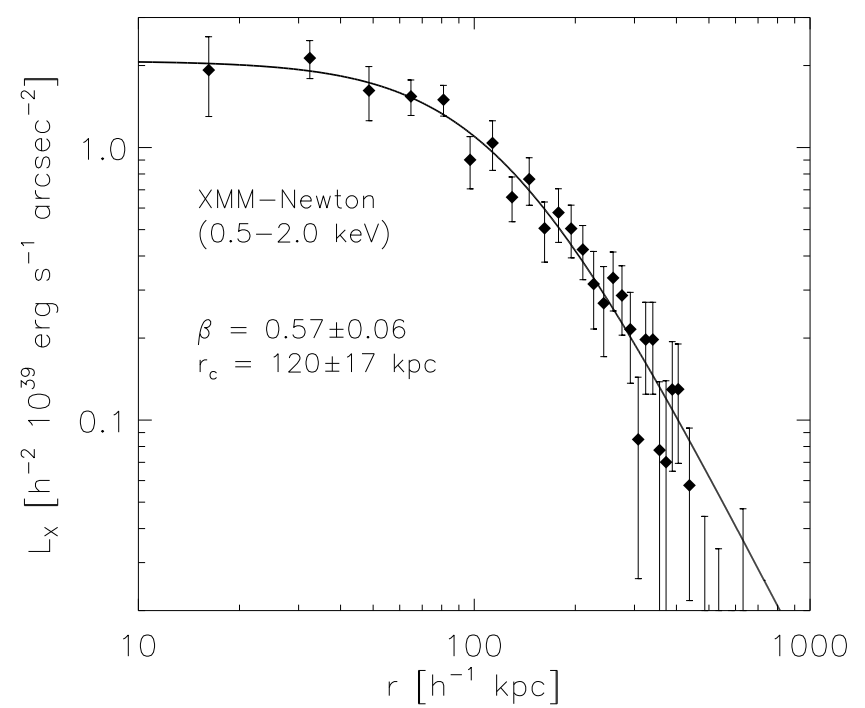

Fig. 10. Best-fit $\beta$-model for the X-ray halo. A cooling flow is absent.

than those of the other three fossil groups presented by the same authors.

We mention here that the X-ray halo of E0454 was detected previously and is listed as object \#6 in the Chandra cluster sample of Boschin (2002). The reported centroid of the X-ray flux is located $\sim 41 \pm 8 \mathrm{kpc}$ south-east of E0454, whereas the XMM-Newton data reveals only a small offset of $24 \pm 16 \mathrm{kpc}$ to the North-West. We explain this by the fact that XMM-Newton collected more than 10 times as many photons as Chandra.

\section{Weak lensing analysis}

The strength of a gravitational lens scales with the ratio of the angular diameter distances $D\left(z_{1}, z_{2}\right)$ between the lens and the source and between the observer and the source. The more distant the source the stronger the lensing effect, but for a lens redshift $z_{l}=0.26$ and sources at $z_{s}>0.8$ it is effectively constant. One must project the sources to some arbitrarily chosen reference redshift $\left(z_{r}=1\right)$ and rescale the shear estimator (the image ellipticities) accordingly to obtain comparable shear values,

$\varepsilon_{1 / 2}=\varepsilon_{1 / 2}^{0} \frac{D\left(z_{l}, z_{r}\right)}{D\left(0, z_{r}\right)} \frac{D\left(0, z_{s}\right)}{D\left(z_{l}, z_{s}\right)}$

This rescaling decreases (enhances) the noise for $z_{s}>z_{r}\left(z_{s}<z_{r}\right)$ and is taken into account by individual weighting factors

$w=\left(\frac{D\left(z_{l}, z_{s}\right)}{D\left(0, z_{s}\right)} \frac{D\left(0, z_{r}\right)}{D\left(z_{l}, z_{r}\right)}\right)^{2}$

Before we could proceed on the weak lensing analysis of J0454 we had to remove the lensing contribution of MS0451 from the data by subtracting a singular isothermal sphere (SIS) tangential shear profile parametrised with $\sigma_{v}=1354 \mathrm{~km} \mathrm{~s}^{-1}$. This value was taken from Carlberg et al. (1997), who used an iterative outlier rejection process for its determination. A concrete error estimate was not given, but by comparing to other measurement methods in their work, we adopted an uncertainty of 5\%. Other known structures apart from MS0451 (see Fig. 6) do not need to be taken into account, as their angular separation is too large and their velocity dispersion is too low to leave a measurable footprint at the position of J0454. The X-ray data are consistent 
with this picture, revealing no structures apart from MS0451 that could add discernible lensing signals to J0454.

\subsection{Mass reconstruction}

We use the finite-field method from Seitz \& Schneider (2001) to reconstruct the projected surface mass density, $\kappa$, from the sheared images. This method uses the field border as a boundary condition, which makes reconstructions of non-rectangular areas difficult. We therefore work on a 16!'8 wide rectangle inscribed into the HST/ACS mosaic. Our code is freely available ${ }^{3}$ and based on the original version from Seitz \& Schneider (2001).

The convergence $\kappa$ is determined up to an additive constant, the "mass-sheet" degeneracy, which is safely broken by assuming that $\kappa$ vanishes on average along the border of the field. The algorithm only works for under-critical regions with $\kappa<1$, i.e. strong lensing areas are not reconstructed reliably. In the case of J0454 this affects only the innermost 4" (see Sect. 7), which is well below the resolution limit and thus of no concern.

The resulting density map must not be interpreted without a corresponding noise map. For example, bright stars cause holes in the data field, which locally increase the noise due to the reduced number density of galaxies. In addition, the smoothing length for the shear field must be larger than these holes. Otherwise, the boundary condition of a rectangular data field is violated, resulting in a corrupted solution. To obtain the noise map, we created 1000 realisations of randomised galaxy orientations keeping their positions fixed, and obtained $\kappa$ for each. The two-dimensional rms of these $\kappa$-maps yields the desired noise map. Since lensing increases the ellipticities of galaxies, we removed the SIS shear profile of J0454 (Sect. 6.2) from the data prior to the randomisations. Otherwise the noise at the cluster position would be overestimated.

The $S / N$-level of the mass map is shown in Fig. 9. J0454 is detected on the $4.7 \sigma$ level with a peak convergence of $\kappa=0.20$. It is the only significant $(S / N>4)$ mass peak besides MS0451 $(S / N=7.7)$, and located $12 \pm 5^{\prime \prime}$ south of E0454. The uncertainty in the position was determined from boot-strapping the shear catalogue. The mass of J0454 within $182 \mathrm{kpc}$ (approximately tracing the $S / N=1$ contour $)$ is $M=(0.38 \pm 0.09) \times$ $10^{14} M_{\odot}$. This is not comparable to $M_{200}$ since it is integrated within a much smaller radius. A determination of $M_{200}$ from the reconstructed density map is not sensible as the noise entirely dominates the signal in the larger aperture. However, we can infer a lower limit of $r_{200} \gtrsim 650 \pm 50 \mathrm{kpc}$. One way to test the integrity of the detection is to check for noise peaks in the 1000 randomisations with equal or higher significance. No such peak is found, consistent with the expectation $(0.21$ peaks $)$ from idealised Gaussian noise. In reality the noise is non-Gaussian as the dispersion of image ellipticities is non-Gaussian. Probing the actual differences for $\sim 5 \sigma$ peaks would require many more randomisations, but would not change our main conclusion here that is that we detected a real signal.

As mentioned previously, we removed the contribution of MS0451 by subtracting a SIS profile with $\sigma_{v}=1354 \mathrm{~km} \mathrm{~s}^{-1}$. Changing this value by $5 \%$ alters the mass estimate by $0.1 \%$, hence this measurement is insensitive to the presence of MS0451. This is not unexpected as the separation between J0454 and M0451 is large and $\kappa$ is a local quantity, resulting in no overlap of the clusters' projected surface mass densities.

\footnotetext{
3 http://www . astro.uni-bonn.de/ mischa/download/ massrec.tar
}

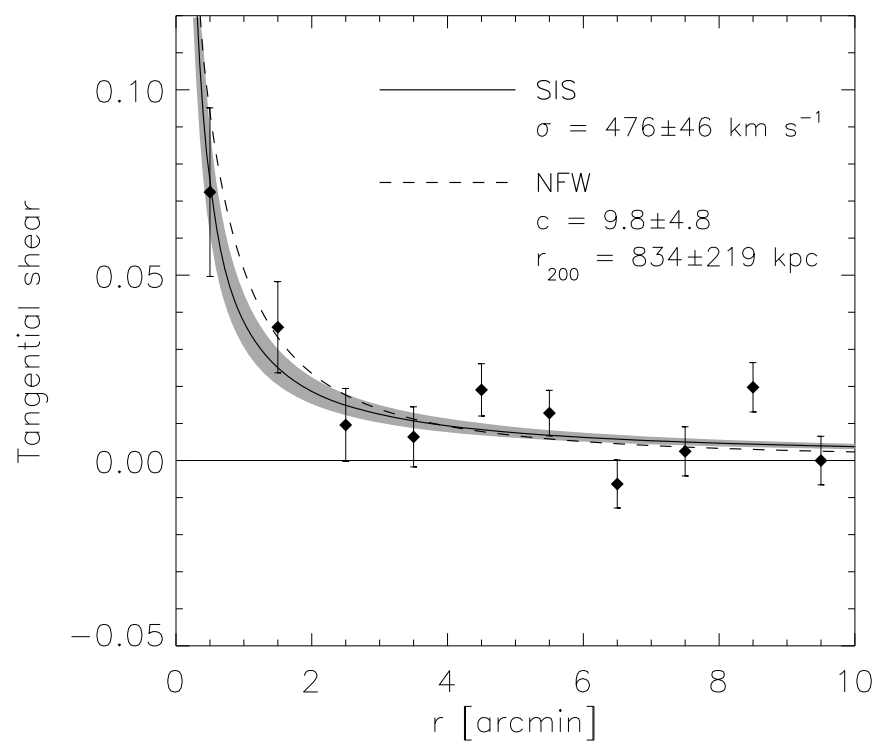

Fig. 11. Tangential shear of J0454 and best-fit SIS and NFW profiles. The shaded area shows the $68 \%$ confidence region of the SIS fit.

\subsection{SIS and NFW fits to the tangential shear profile}

We fit SIS and NFW profiles to the tangential shear around J0454 (Fig. 11), assuming a spherical symmetric density distribution. As compared to the mass reconstruction, the results are not model-independent. For the SIS we furthermore assumed that the system is in virial equilibrium with isotropic distribution of the orbits, having a density profile

$\rho_{\mathrm{SIS}}(r)=\frac{\sigma_{v}^{2}}{2 \pi G r^{2}}$

which yields, in analogy to the derivation of Eq. (14),

$r_{200}=\frac{\sqrt{2}}{10} \frac{\sigma_{v}}{H(z)}$

(note the different pre-factor).

The tangential shear is measured with respect to a reference point, which should be near or at the centre of mass, depending on substructure. We identify the position where the tangential shear is maximised with a matched-filter technique (the $S$-statistics or peak finder, see Schirmer et al. 2007). The signal is maximised for a 5.5 wide filter approximating the NFW shear profile, detecting J0454 on the $5.2 \sigma$ level $12^{\prime \prime} \pm 5^{\prime \prime}$ southeast of E0454 (position angle $162 \pm 2$ degrees, both error estimates from bootstrapping). This is coincident with the peak of the mass reconstruction and indicates a robust choice for the reference point. In general, the two peaks would not necessarily coincide as both methods compute very different quantities. Deviations can occur in particular for clusters with significant substructure (see e.g. Holhjem et al. 2009), provided that the $S / N$ is high enough to resolve such features. With this reference point the SIS fit yields $\sigma_{v}^{\mathrm{wl}}=476 \pm 46 \mathrm{~km} \mathrm{~s}^{-1}$ and $M_{200}=(0.90 \pm 0.26) \times 10^{14} M_{\odot}$, and from the NFW fit we obtained a concentration parameter of $c=9.5 \pm 4.8, r_{200}=834 \pm$ $219 \mathrm{kpc}$ and $M_{200}=(0.84 \pm 0.66) \times 10^{14} M_{\odot}$. Contrary to the convergence $\kappa$, the shear is a non-local quantity and therefore more susceptible to changes in the velocity dispersion assumed for MS0451. For example, decreasing (increasing) its $\sigma_{v}$ by $5 \%$ results in a $2 \%(4 \%)$ increase of the velocity dispersion for J0454. These effects are included in our error budget. 


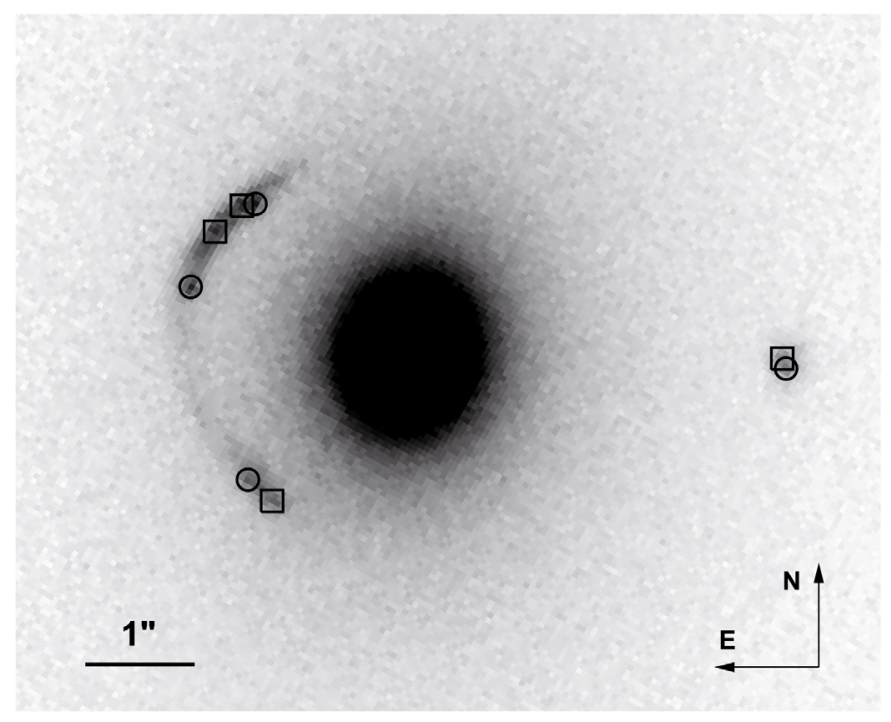

Fig. 12. HST/ACS image of the strong lens. The counter image and the arcs reveal two maxima in the source intensity distribution, forming two sets of multiple image systems. They are marked by circles and squares and are used for the lens modelling.

To quantify the effect of possible errors in the choice of the reference point, we repeated the analysis using the core of E0454 and the centroid of the distribution of elliptical galaxies within $r_{200}=830 \mathrm{kpc}$. This yielded $\sigma_{v}=462 \pm 49 \mathrm{~km} \mathrm{~s}^{-1}$ and $376 \pm 58 \mathrm{~km} \mathrm{~s}^{-1}$, respectively. The first fit is qualitatively slightly worse than the original fit but still acceptable, whereas the second is significantly deteriorated. The centroid of the distribution of elliptical galaxies can therefore be ruled out as the centre of mass. We show below based on strong lensing that this also applies to E0454.

\section{Strong lensing analysis}

\subsection{Lens modelling}

We identified two sets of multiple image systems, corresponding to two bright knots in the source intensity distribution and identified by circles and squares in Fig. 12. The lens is modelled using a pseudo-isothermal elliptic mass distribution (PIEMD, Kassiola \& Kovner 1993, with zero core radius),

$\kappa\left(\theta_{1}, \theta_{2}\right)=\frac{b}{1+q}\left(\theta_{1}^{2}+\frac{\theta_{2}^{2}}{q^{2}}\right)^{-1 / 2}$,

where $b$ is the strength and $q$ is the axis ratio. The $1 /(1+q)$ normalisation is needed to match the profile in Kassiola \& Kovner (1993) that was defined using ellipticities $(\epsilon \equiv(1-q) /(1+q))$ instead of axis ratios. The distribution is translated by the centroid position and rotated by the position angle, $\mathrm{PA}_{\mathrm{L}}$. Furthermore, we allowed a constant external shear with strength $\gamma_{\text {ext }}$ and $\mathrm{PA}_{\text {ext }}$. In total, there are 11 parameters: 4 for the two source positions, 5 for the PIEMD, and 2 for the external shear. The two sets of multiple images provide 16 constraints. Note that the modelling is independent of lens and source redshifts.

We used the strong lens modelling code (Halkola et al. 2010, in preparation) based on Halkola et al. (2006), Halkola et al. (2008), Suyu et al. (2006) and Dunkley et al. (2005). Markov chain Monte Carlo (MCMC) methods were employed to obtain the posterior probability distributions of the lens parameters. We placed Gaussian priors on the centroid, $q$, and $\mathrm{PA}_{\mathrm{L}}$ of
Table 3. Marginalised strong lens parameters.

\begin{tabular}{lc}
\hline \hline$\gamma_{\text {ext }}$ & $0.12 \pm 0.02$ \\
$\mathrm{PA}_{\mathrm{ext}}$ & $91_{-3}^{+5}[\mathrm{deg}]$ \\
\hline$b$ & $2.38_{-0.05}^{+0.03}[\operatorname{arcsec}]$ \\
$q$ & $0.80_{-0.08}^{+0.06}$ \\
$\mathrm{PA}_{\mathrm{L}}$ & $161_{-6}^{+9}[\mathrm{deg}]$ \\
\hline
\end{tabular}

Notes. The position angles (PA) are counted from North to East. The uncertainties on the parameters correspond to the $68 \%$ posterior credible interval.

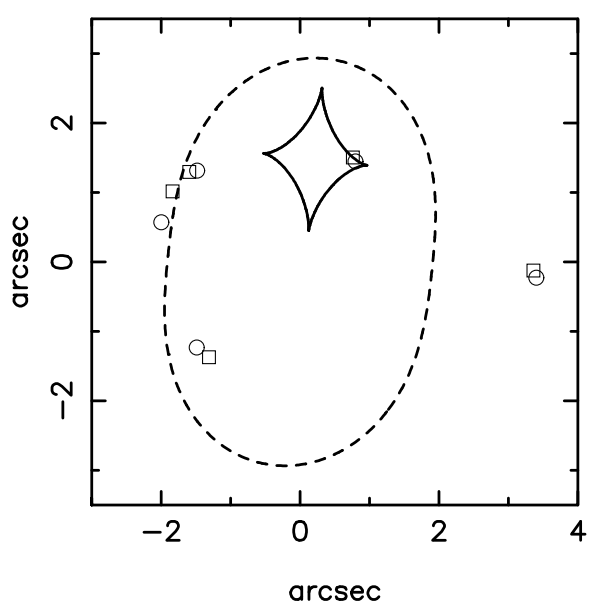

Fig. 13. The most probable critical curve (dashed) and caustic curve of the lens. The caustic consists of four folds (solid lines) joining at four cusps. The modelled source and image positions for the two sets of multiple-image systems are marked by squares and circles.

the PIEMD (with Gaussian widths of $0.05^{\prime \prime}, 0.09$ and $10^{\circ}$, respectively) based on the observed light distribution.

Table 3 lists the results of the marginalised lens parameters from a MCMC chain of length $10^{5}$ after the burn-in phase. Typical predicted image positions agree with the observations within 1 pixel (rms $\sim 00^{\prime} 03$ ). Figure 13 shows the critical and caustic curves of the most probable lens parameters. The arc is in a fold configuration, close to being in a cusp configuration (i.e., the positions of the bright knots in the source lie next to a fold and are in the vicinity of a cusp of the caustic curves). The two merging images form the northern half of the arc with magnifications $\mu=8.2-33.8$, and the other image the southern arc with $\mu=3.0-4.4$. The counter image has $\mu \sim 2.3$.

The separations between the arc and the lens, and between the counter image and the lens, are 1".85-2'. 18 and $33^{\prime \prime} 42$, respectively. This asymmetry requires the presence of significant external shear, $\gamma_{\mathrm{ext}}=0.12$. As gravitational lensing is an achromatic process, all images should have similar colours, which allowed us to test the counter image hypothesis. Since the lensed images are very near the core of E0454 we subtracted a model for the lens galaxy light before obtaining usable photometry. Thereto we fit an elliptic Sersic model to the $u^{*} B V R I z$ data using GALFIT (Peng et al. 2002). The resulting images and source fluxes are shown in Figs. 14 and 15. We found good agreement confirming the lens modelling. Only the $V$-band flux of the southern arc appears too low, which is a consequence of the worse seeing in this filter and the fact that this image is closest to the lens making it very susceptible to over-subtraction effects. 

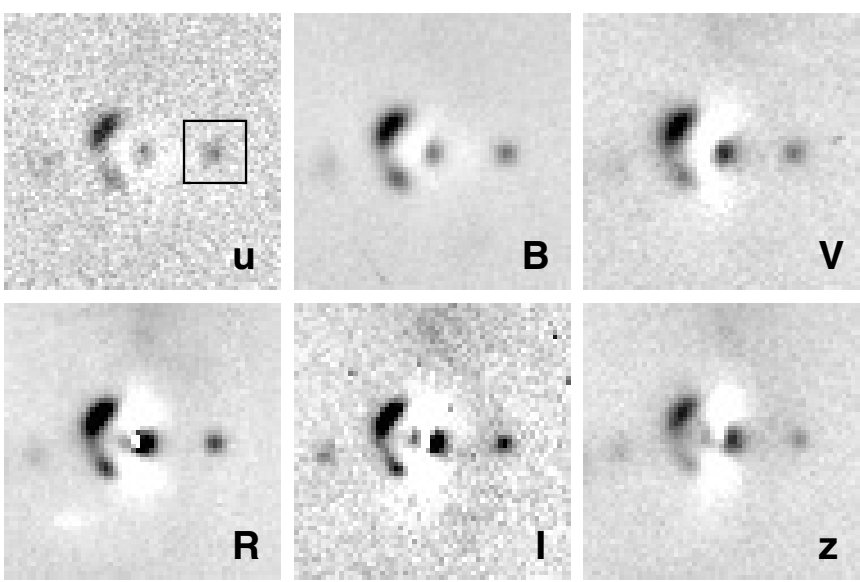

Fig. 14. The arc system after subtracting an elliptic Sersic model from the CFHT $u^{*}$-band and the Subaru BVRIz-band images. The lens is not entirely removed. The counter image is marked with a box. North is up and East is left, the image width is $27^{\prime \prime}$.

\subsection{PIEMD and stellar velocity dispersions}

The equivalent Einstein radius of the PIEMD reads

$\theta_{\mathrm{E}}^{\mathrm{PIEMD}}=2 b \frac{\sqrt{q}}{1+q}$

(e.g. Koopmans et al. 2006) and evaluates to 2 '. $37 \pm 0$.'04. These authors have also shown that $\theta_{\mathrm{E}}^{\mathrm{PIEMD}}$ corresponds to that of a classical spherically symmetric SIS,

$\theta_{\mathrm{E}}=4 \pi\left(\frac{\sigma_{v}}{c}\right)^{2} \frac{D_{l s}}{D_{s}}$

yielding a PIEMD velocity dispersion of $\sigma_{v}^{s l}=319 \pm 4 \mathrm{~km} \mathrm{~s}^{-1}$ for a source redshift of $z_{s}=2.1 \pm 0.3$.

Elíasdóttir et al. (2007) provide a recipe through which the PIEMD strong lensing velocity dispersion can be linked to the observed stellar velocity dispersion $\sigma^{*}$. Their mass model is a parametrised truncated PIEMD (hereafter: dPIE) with core radius $a$ and scale radius $s$, which becomes identical to our model in the limit of $a \rightarrow 0$ and $s \rightarrow \infty$. We obtain

$\sigma_{\mathrm{dPIE}}=\left(\frac{2}{3} \frac{c^{2}}{4 \pi} \frac{D_{s}}{D_{l s}} \theta_{\mathrm{E}}^{\text {PIEMD }}\right)^{1 / 2}=260 \pm 4 \mathrm{~km} \mathrm{~s}^{-1}$.

The relation between $\sigma_{\text {dPIE }}$ and $\sigma^{*}$ is shown in Fig. 20 of Elíasdóttir et al. (2007). Our PIEMD corresponds to their asymptotic limit, approximated by the solid line in that figure. The radius $R$ (3 FORS2 detector rows or 0.' 6 ) within which we measured $\sigma^{*}$ is significantly smaller than their effective radius $R_{\mathrm{e}}$ (half mass radius), hence $R / R_{\mathrm{e}} \ll 1$. We thus expect $\sigma^{*}$ to be between $0.95 \sigma_{\mathrm{dPIE}}$ and $1.15 \sigma_{\mathrm{dPIE}}$ or $250-300 \mathrm{~km} \mathrm{~s}^{-1}$. A fit of the Doppler-broadened $\mathrm{NaD}$ absorption doublet yields $\sigma^{*}=210 \pm$ $80 \mathrm{~km} \mathrm{~s}^{-1}$, indicating that E0454 possibly does not contain all the lensing mass. Up to $50 \%$ could be located in the halo of J0454 (see also McKean et al. 2010, for a similar example), but the large error bars do not allow a definite conclusion.

These considerations depend on the arc redshift which could not be determined unambiguously from spectroscopy (see Sect. 2.3). For $z_{\mathrm{arc}}=0.4(1.0)$ we would have $\sigma_{\mathrm{dPIE}}=$ $515 \mathrm{~km} \mathrm{~s}^{-1}\left(345 \mathrm{~km} \mathrm{~s}^{-1}\right)$ and similarly high stellar velocity dispersions. Thus $z_{\text {arc }}=0.4$ is clearly ruled out by the velocity dispersion measured $\left(\sigma^{*}=210 \pm 80 \mathrm{~km} \mathrm{~s}^{-1}\right)$, and also by the fundamental plane properties of BCG galaxies

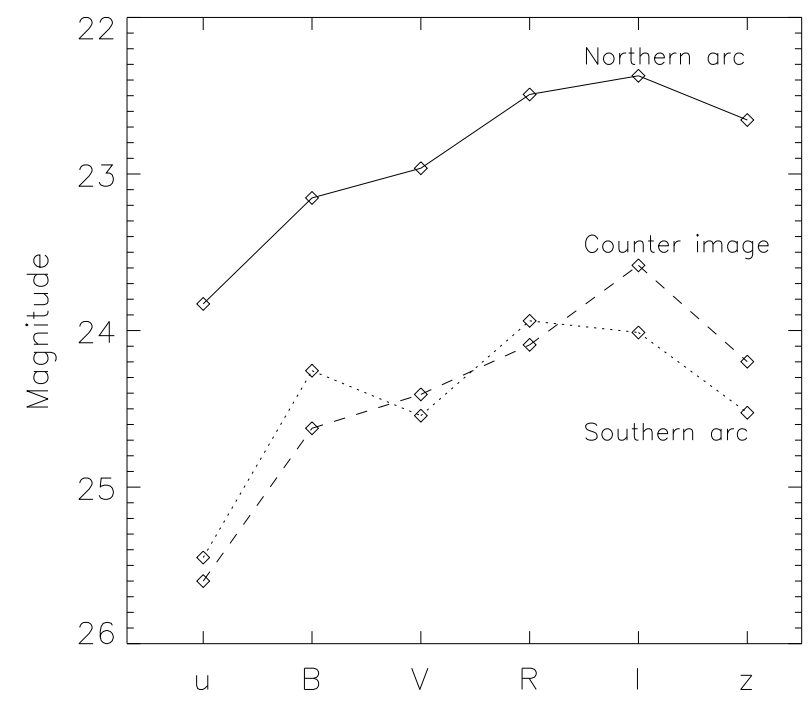

Fig. 15. Magnitudes of the arc components and the counter image, obtained from the images in Fig. 14. The uncertainties in the values are of the order of 0.2 mag due to the residuals from the removal of E0454.

(Desroches et al. 2007). The latter predict $\sigma^{*}=280 \pm 35 \mathrm{~km} \mathrm{~s}^{-1}$ for a BCG with the absolute I-band luminosity of E0454 $(-24.1 \mathrm{mag})$. A redshift of $z_{\text {arc }} \sim 1.0$ would still be permitted within $2 \sigma$ errors, but the redshift most consistent with the data are $z_{\text {arc }}=2.1 \pm 0.3$, as assumed throughout our paper.

\section{Interpretation and discussion}

\subsection{E0454 is not at the centre of the dark matter potential}

Strong gravitational lensing by galaxy groups is very sensitive to the local group environment, and in particular to the internal distribution of dark matter (Momcheva et al. 2006). During the build-up of the morphology-density relation individual dark matter haloes get partially stripped and integrated into the group halo. In these systems, strong lensing can occur by individual galaxies with typical Einstein radii of $\theta_{\mathrm{E}}=1^{\prime \prime}-2^{\prime \prime}$, but also by the common and more massive group halo with $\theta_{\mathrm{E}}=3^{\prime \prime}-8^{\prime \prime}$ (see e.g. Fassnacht et al. 2008; Limousin et al. 2009).

The Einstein radius for the $476 \pm 46 \mathrm{~km} \mathrm{~s}^{-1}$ weak lensing SIS halo of J0454 and a source redshift of $z_{s}=2.1 \pm 0.3$ is

$\theta_{\mathrm{E}}=4 \pi\left(\frac{\sigma_{v}}{c}\right)^{2} \frac{D_{l s}}{D_{s}}=5.28 \pm 1^{\prime \prime} .02$,

more than twice as large as the observed arc radius of $2 . \prime 37$. Hence the strong lens effect is caused by E0454 and not by the more massive group halo. Given that all masses along the line of sight contribute to the lensing potential, the immediate consequence of this observation is that E0454 cannot be, on a $3 \sigma$-level, located at the centre of the gravitational potential of J0454. Otherwise the arc radius would be significantly larger.

This argument would not hold anymore if the lensed source was at very low redshift (we assume that it is at high redshift, see Sects. 2.3 and 7.2). The Einstein radius of 2 '. 37 would be reproduced for $z_{s}=0.42$, but already for slightly higher redshifts such as $0.5(0.6)$ it would increase to 2 ". 93 (3". 45). But even if we overestimated the source redshift significantly, E0454 could still not be located at J0454's halo centre as we show in the following using the lensed image configuration.

The lens modelling (Table 3 ) requires a large amount of external shear, $\gamma_{\mathrm{ext}}=0.12 \pm 0.02$, which can be caused by a 


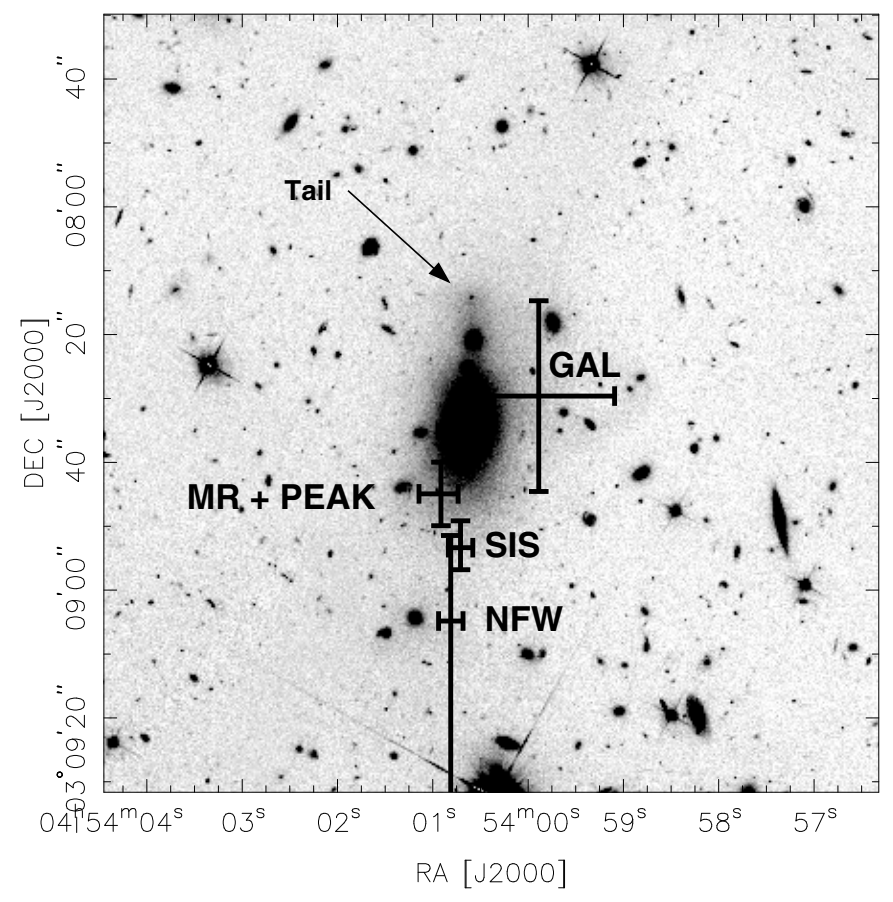

Fig. 16. The various estimates for the halo centre of J0454: distribution of elliptical galaxies, mass reconstruction and peak finder, and from the external shear coming from an SIS or NFW profile. The arrow marks a tidal feature in the optical halo of E0454.

different lens along the line of sight, but also by an offset of E0454 in the halo of J0454. The SIS model for the background cluster MS0451 predicts $\gamma_{\mathrm{ext}}=0.021$ with PA $=108$ degrees. To obtain the external shear required, we need additional components whose net shear is $\gamma_{\mathrm{ext}}=0.100 \pm 0.017$ and $\mathrm{PA}=86.5 \pm$ 0.6 degrees. Since there is no evidence for other suitable lenses in the Keck spectra and the XMM-Newton data, this signal must come from $\mathrm{J} 0454$ alone. Its centre of mass must be located $22^{\prime \prime} \pm 4^{\prime \prime}(89 \pm 16 \mathrm{kpc})$ south of E0454 for a SIS profile, and $31_{-12}^{+30} \operatorname{arcsec}\left(126_{-49}^{+122} \mathrm{kpc}\right)$ for NFW (see Fig. 16). The same external shear could also be caused if the halo was at identical distances to the North of E0454, but this is ruled out at the $4 \sigma$ level by the mass reconstruction and the peak finder, both of which locate the centre of mass $12^{\prime \prime} \pm 5^{\prime \prime}$ south of E0454. This is unlikely to be the real centre, as neither the mass reconstruction nor the peak finder are able to resolve such substructures in the halo for the given lensing signal-to-noise ratio. A much larger number density of lensed background galaxies than $n=73 \operatorname{arcmin}^{-2}$ would be required for this purpose.

The different offsets predicted by the spherically symmetric NFW and SIS density profiles are obviously modeldependent. Furthermore, an elliptical halo would increase the offset if the halo's projected major axis pointed to E0454. The reason for this is the increased projected mass and thus shear seen by the strongly lensed light bundle towards J0454, putting the halo at larger separation from E0454 to satisfy the external shear constraint. Likewise a smaller offset would result if the halo minor axis pointed towards E0454. The first scenario is more likely as the distribution of red sequence galaxies is significantly elongated North-South within $r_{200}$ (see Fig. 5), which is expected if the galaxies are virialised within an elliptic halo. In addition, E0454 is elongated along the same direction, and Okumura et al. (2009) show that the central luminous red galaxies in clusters are preferentially aligned within $\sim 35$ degrees with their host dark matter haloes. The separations between E0454 and the halo centre should therefore be regarded as lower limits.

\subsubsection{Effect of sub-haloes on the external shear}

Individual galaxies can significantly affect strong lensing systems in cluster environments. We modelled the red sequence galaxies with SIS profiles to estimate their contribution to the external shear, using the velocity dispersions predicted by the Faber-Jackson relation from Desroches et al. (2007). Including successively more galaxy haloes going from the strong lens outwards, we find that the external shear increases gradually and stabilises at $\gamma_{\text {ext }}=0.036 \pm 0.056$ with PA $=76$ degrees. Only 5 galaxies within 0.51 contribute to the signal. The uncertainty in the shear is large and based on the intrinsic scattering of the Faber-Jackson relation. If we systematically increase the predicted velocity dispersions of all 5 galaxies by the $1 \sigma$ range allowed by Faber-Jackson, then the entire external shear can be explained by these haloes. In the other extreme, by lowering the velocity dispersion by $1 \sigma$, the contribution to the external shear becomes zero.

We therefore expect halo substructures to contribute about $30 \%$ to the total external shear. Since this lowers the shear coming from a smooth common group or cluster halo with $\sigma \sim$ $480 \mathrm{~km} \mathrm{~s}^{-1}$, the offset from E0454 to the centre of this halo becomes larger. For example, in case of a SIS halo the separation would increase from $22^{\prime \prime}$ to $40^{\prime \prime}$. The position angle, i.e. the location of the halo centre south of E0454, remains unchanged. We ignore the smaller effects of substructure for the rest of the paper as the main result, i.e. the presence of an offset of E0454 with respect to the centre of the projected mass distribution, remains unaffected.

\subsubsection{Interpretation: a group falling into a cluster, or a filament collapsing onto a group?}

How can it be explained that E0454, which is significantly more luminous and massive than all other member galaxies, is not located at the minimum of the gravitational potential? Such offsets are not uncommon for normal groups and clusters (Oegerle \& Hill 2001; von der Linden et al. 2007; Skibba et al. 2010), but for old and evolved fossils they are unusual and have not been reported previously. If all galaxies in J0454 had the same origin, then the observations are difficult to reconcile. A simple solution would be that E0454 formed outside of J0454 in a separate small group which is now falling into J0454. This could also explain the velocity offset of $+240 \mathrm{~km} \mathrm{~s}^{-1}\left(+540 \mathrm{~km} \mathrm{~s}^{-1}\right)$ observed between the BCG and the population of elliptical (spiral) galaxies (see Sect. 4.2 and Zabludoff \& Mulchaey 1998; Oegerle \& Hill 2001). We present more support for this scenario below based on the properties of the X-ray halo.

There is one observational difficulty in this picture. If the halo into which the group is falling represents a fully formed NFW mass distribution, then one would expect a galaxy or concentration of galaxies at its centre whose brightness is reasonably scaled to the halo mass. However, no such galaxies are seen. From this we conclude that the halo is not fully assembled yet, or decomposed into several sub-haloes in a filament projected along the line of sight, mimicking a spherical system. In the latter case, galaxies could be streaming along the filament onto the denser fossil group, presenting an alternative interpretation of the system (B. Fort, private communication). Unfortunately neither the strong nor the weak lensing data allows us to 
distinguish between a filament and a more spherical cluster. We pool both scenarios under the term "infall hypothesis", indicating a fossil group still forming an object of its own in a larger system (J0454).

\subsection{X-ray halo properties support infall hypothesis}

Whereas the strong lensing data require an offset of $90-120 \mathrm{kpc}$ between E0454 and the halo of J0454, only a weakly significant offset of $24 \pm 16 \mathrm{kpc}$ exists between E0454 and the X-ray halo. The latter appears to be gravitationally bound by E0454 and not by $\mathrm{J} 0454$. Even though the X-ray halo overlaps in projection with the presumed core of J0454, no significant mass transfer has happened yet as the X-ray halo appears undisturbed. This can be explained if E0454 still forms a local minimum and thus a system of its own in the larger gravitational potential of J0454. The significantly lower X-ray mass of $0.34 \times 10^{14} M_{\odot}$ as compared to $(0.75-0.90) \times 10^{14} M_{\odot}$ from galaxy counts and weak lensing supports this interpretation. The same holds for the $\beta$-model velocity dispersion $\left(316 \pm 26 \mathrm{~km} \mathrm{~s}^{-1}\right)$ which matches the strong lensing value $\left(319 \pm 4 \mathrm{~km} \mathrm{~s}^{-1}\right)$ much better than the one derived from weak lensing $\left(476 \pm 46 \mathrm{~km} \mathrm{~s}^{-1}\right)$. The halo temperature of $1.1 \mathrm{keV}$ is also more characteristic for a group with $\sim 330 \mathrm{~km} \mathrm{~s}^{-1}$ than the optically determined overall $480 \mathrm{~km} \mathrm{~s}^{-1}$. If E0454 indeed represents a very evolved and virialised former group of galaxies that is now falling into J0454 (Sect. 8.1), then one would expect the X-ray properties to reflect a less massive and smaller system than J0454. Given the undisturbed X-ray halo, the absence of shock fronts and the low temperature, we presume that E0454 has not yet passed through J0454 and is thus inbound for the first time. The present data does not allow us to infer more information about the three-dimensional orientation of the trajectory. To this end we would need a better sampling of the peculiar motions of the member galaxies in J0454.

\subsection{X-ray offsets in other strong lensing or fossil groups}

The offset of $24 \pm 16 \mathrm{kpc}$ between the X-ray centroid and E0454 is consistent with those of other groups. Four of the strong lensing selected systems by Fassnacht et al. (2008) have X-ray haloes, coinciding within 25-50 $h_{100}^{-1} \mathrm{kpc}$ with the brightest group galaxy (BGG). Even smaller offsets have been observed for the five fossil groups in Khosroshahi et al. (2006b), where the X-ray centroids of four systems match those of the BGGs. For the fifth system, RX J1552.2+2013, an offset of $12 \mathrm{kpc}$ is reported, but the authors argue that it is unlikely to be real. Fossil samples that were cross-matched with the ROSAT AllSky Survey (Voges et al. 1999) such as those by Santos et al. (2007) and La Barbera et al. (2009) show larger offsets of up to 50 and $90 \mathrm{kpc}$, respectively. These should be interpreted with caution though due to the poor angular resolution of ROSAT. In general, X-ray selected galaxy groups often show small offsets, but in dynamically disturbed or merging systems they can become larger than $100 \mathrm{kpc}$ (Jeltema et al. 2006, 2007).

Whether small X-ray offsets are representative for strong lensing X-ray groups is currently difficult to answer due to the small sample size and possible selection effects. For example, the groups from Fassnacht et al. (2008) could be biased towards systems for which the X-ray centroid and the BGG coincide, as this would boost the central density giving rise to strong lensing. On the other hand, groups with more complicated dynamical states (and larger X-ray offsets) have increased lensing crosssections and should therefore be selected as well.

\subsection{Comparison with other strong lensing groups}

Limousin et al. (2009) use an automated algorithm to detect strong lensing features. They were looking for Einstein radii larger than $3^{\prime \prime}$, targetting group-scale strong lenses, and found 13 such systems. The authors also report weak lensing measurements of the velocity dispersions in the range of $500-800 \mathrm{~km} \mathrm{~s}^{-1}$. A comparison of the weak and strong lensing Einstein radii is not made, but the according values have been tabulated. In general there seems to be good agreement between the two estimates if the group halo is responsible for the lensing. About half of the groups show significantly larger strong lensing Einstein radii, most likely systems where the lensing has been boosted by the potential of an individual galaxy in addition to the group halo. Due to the lower cut-off in $\theta_{\mathrm{E}}$ objects like J0454 with large weak and small strong lensing Einstein radii are filtered out. A survey aiming at smaller strong lensing features near the core of the BGG could identify systems similar to J0454. In combination with a high external shear this would be a prime indicator for substructure and a possible infall.

\subsection{Spatial and dynamic misalignment of E0454}

More evidence for the infall hypothesis arises when looking at the sample of seven groups selected by Fassnacht et al. (2008) for their strong lensing effects. These authors found that the BGG almost always coincides with the spatial and the dynamical group centre. E0454 on the other hand is marginally consistent within $1 \sigma$ with the centre of the distribution of elliptical galaxies. In addition, its velocity deviates by $2.5 \sigma$ (half the velocity dispersion) from the mean recession velocity of the ellipticals, and even more so from that of the spiral galaxies (comparable to the velocity dispersion, see Sect. 4.2). E0454 also contradicts the nine X-ray selected groups and poor clusters of Mulchaey et al. (2006), who found that BGGs coinciding with the X-ray centroid have the same mean recession velocity as the surrounding group. It would be worthwhile to look for similar deviations in the currently existing samples of fossil groups.

With a more complete sampling of velocities of the elliptical galaxies we could analyse these deviations in more detail, possibly identifying a dynamic sub-population of galaxies belonging to the fossil group (or, if we summon our alternative interpretation, identify galaxies in the filament streaming towards the group). With the data at hand we cannot estimate how many galaxies comprise the fossil group. A tidal feature in E0454's optical halo (see Fig. 16) indicates that the accretion process in the fossil component of J0454 has not yet finished, and therefore it is plausible that E0454 is not the only galaxy belonging to that component.

\subsection{Dynamic disturbances, $X$-ray offsets and cooling flows}

Dynamically disturbed haloes can suppress or reheat cooling cores, as has been shown by Sanderson et al. (2009) for the 65 systems in the Local Cluster Substructure Survey (LoCuSS, median redshift $z=0.23$ ). They demonstrated that for clusters without cooling core or with inactive BCGs the probability distribution function of the projected offset between the X-ray centroid and the BCG peaks between 40 and $60 \mathrm{kpc}$. Conversely, cooling core clusters never showed offsets larger than $15 \mathrm{kpc}$. With an offset of $24 \mathrm{kpc}$ and no traces of star formation in the FORS2/VLT spectrum, E0454 matches the LoCuSS observations. In addition there are also hints for dynamic disturbances. E0454 is embedded in an extended optical halo (see Fig. 16), 
Table 4. Summary of the main results.

\begin{tabular}{llll}
\hline \hline Method & $\sigma_{v}\left[\mathrm{~km} \mathrm{~s}^{-1}\right]$ & $r_{200}[\mathrm{kpc}]$ & $M_{200}\left[10^{14} M_{\odot}\right]$ \\
\hline Galaxy counts & - & $\mathbf{8 1 1} \pm \mathbf{4 6}$ & $0.74 \pm 0.15$ \\
Spectr. (early type) & $\mathbf{4 8 0} \pm \mathbf{2 0}$ & $1054 \pm 44$ & $1.69 \pm 0.14$ \\
Spectr. (late type) & $\mathbf{5 9 0} \pm \mathbf{2 0}$ & $1295 \pm 44$ & $3.14 \pm 0.21$ \\
X-ray ( $\beta$-model) & $\mathbf{3 1 6} \pm \mathbf{2 6}$ & $617 \pm 28$ & $0.34 \pm 0.10$ \\
Weak lens. (SIS) & $\mathbf{4 7 6} \pm \mathbf{4 6}$ & $853 \pm 82$ & $0.90 \pm 0.26$ \\
Weak lens. (NFW) & - & $\mathbf{8 3 4} \pm \mathbf{2 1 9}$ & $0.84 \pm 0.66$ \\
Weak lens. (MR) & - & $650 \pm 50^{*}$ & $\mathbf{0 . 3 8} \pm \mathbf{0 . 0 9}$ \\
Strong lensing & $\mathbf{3 1 9} \pm \mathbf{4}$ & $700 \pm 9$ & $0.50 \pm 0.01$ \\
\hline
\end{tabular}

Notes. Values in bold face are the primary measurements, the others were derived from these.

${ }^{(*)}$ The mass estimate from the weak lensing mass reconstruction is obtained within a radius of $182 \mathrm{kpc}$, significantly smaller than $r_{200}$. The given values are therefore lower limits.

forming a tidal tail in the North at a distance of about $90 \mathrm{kpc}$ from the core. This feature is too small and too localised to be attributed to a current major merger event. It could be a residual of a recently disrupted small companion galaxy, or caused by tidal disturbances of galaxies orbiting very close to the halo (objects \#2, 3 and 4 from Table A.1). A similar feature is observed in the BCG of the fossil cluster ESO 3060170 (Sun et al. 2004).

E0454 is currently accreting mass, but the rate is too small to have destroyed a previously existing cool core. Burns et al. (2008) have analysed the survival rate of cool cores in merger simulations and found that non-cooling core clusters experienced a high accretion rate with major mergers at $z>0.5$, destroying a potentially existing cool core and also prevent their later reformation. Minor, and in particular late accretion events such as the one observed in E0454 do not suppress a cool core. Thus, if a cooling core were present in E0454, it must have been destroyed at early times. This is consistent with E0454 being a giant elliptical galaxy, as only these galaxies have experienced major mergers in their history (Parry et al. 2009), and fossil systems in particular form their halos at earlier times than other groups (Dariush et al. 2007; von Benda-Beckmann et al. 2008). A systematic survey of fossils regarding cooling flows and cool cores would be helpful in testing theoretical predictions. So far, the number of examined systems is small (see e.g. Sun et al. 2004; Khosroshahi et al. 2004, 2006a) and the amount of fossils with suitable X-ray data poor. This also applies to J0454, for which the data are insufficient to derive a temperature map with sufficient resolution to establish the non-existence of a cooling core.

\subsection{Mass-to-light ratio}

Sheldon et al. (2009a) have obtained mass-to-light ratios for the maxBCG cluster sample in SDSS, using weak lensing to estimate $M_{200}$. The total luminosity within $r_{200}$ was inferred from red sequence galaxies only. To minimise $K$-corrections, it was calculated for the $i$-band bandpass shifted to the median cluster redshift of 0.25 . We adopt their terminology and refer to the shifted bandpass as ${ }^{0.25} i$. A minimum ${ }^{0.25} i$-band luminosity of $10^{9.5} h_{100}^{-2} L_{\odot}$ was required for each galaxy.

From the SIS and NFW fit to the tangential shear profile we obtained an average $\left\langle r_{200}\right\rangle=843 \mathrm{kpc}$, corresponding to $3^{\prime} .47$. Within this radius are 14 and 22 red sequence galaxies with and without spectroscopic confirmation above the minimum luminosity threshold. To correct for the field contamination determined in Sect. 3.1.3, we randomly selected a corresponding number of 5 galaxies from the sample without spectroscopic redshifts and calculated their total flux contribution. This was repeated 100 times to estimate the average background correction. The total luminosity found is $L_{0.25 i}^{\text {tot }}=(6.9 \pm 0.6) \times$ $10^{11} h^{-2} L_{\odot}$, and $M_{200} / L_{0.25 i}=130 \pm 39 h$ for the SIS profile and $M_{200} / L_{0.25_{i}}=122 \pm 96 h$ for NFW. In the rest-frame bandpass the $M / L$ ratios would be $8 \%$ lower. For a cluster with the same number of $N_{200}$ (Sect. 4.3) galaxies as J0454, Sheldon et al. (2009a) predict $\left\langle M_{200} / L_{0.25_{i}}\right\rangle=200 \pm 30 \mathrm{~h}$. Given the scatter present in the luminosity and the mass of a given $N_{200}$ bin (see Sheldon et al. 2009b,a), J0454 does not appear exceptionally over-luminous compared to non-fossil systems. The contribution of the BGG to the total luminosity in $i$-band within $r_{200}$ is $38 \%$.

For completeness we also report the corresponding result for rest-frame $B$-band and the SIS mass, $M_{200} / L_{B}=115 \pm 34 h$. If we include also galaxies bluer than the red sequence, the ratio becomes $101 \pm 30 \mathrm{~h}$. The contribution of the BGG to $L_{B}^{\text {tot }}$ is $34 \%$ for red sequence galaxies alone, decreasing to $29 \%$ if late type galaxies are included. The latter is an upper limit as the sample of late types is incomplete. For the two fossil clusters RX J1416.4+2315 and RX J1552.2+2013 the non-brightest cluster members contribute only $\sim 55 \%$ of the flux of the BCG, i.e. the BCG provides about $2 / 3$ of $L_{B}^{\text {tot }}$ (see Khosroshahi et al. 2007; Cypriano et al. 2006; Mendes de Oliveira et al. 2006).

\section{Summary and conclusions}

In deep ground-based Subaru/Suprime-Cam data we discovered a galaxy strongly lensed by a very bright elliptical galaxy (E0454). Using VLT/FORS2 spectroscopy we confirmed that E0454 is a member of a larger association of galaxies (J0454) at $z=0.26$. The system forms a fossil group with a gap of $2.5 \mathrm{mag}$ in $I$-band between the brightest and the second brightest galaxy within half the virial radius. We have spectroscopically confirmed the membership of 31 galaxies, and furthermore selected 33 objects based on their photometric properties. Our catalogue is complete down to $i \leq 22\left(M_{i}=-18.6\right)$.

The data, being the deepest so far for a fossil group, show that J0454 is a complex system in various stages of mass assembly. Stripping away the layers from outside to inside, we find two filaments extending $4 \mathrm{Mpc}$ from J0454. Within a projected distance of $1.5 \mathrm{Mpc}$ of the centre is a population of spirals with $\sigma_{v}=590 \mathrm{~km} \mathrm{~s}^{-1}$, surrounding a more concentrated and dynamically cooler group of $\sim 50$ galaxies $\left(\sigma_{v}=480 \mathrm{~km} \mathrm{~s}^{-1}\right)$. These form a red sequence with an intrinsic width of $\sigma=0.049$.

Using HST/ACS and photometric redshifts we performed the first weak lensing analysis for a fossil group. The tangential shear profile yields $r_{200} \sim 840 \mathrm{kpc}$ and $M_{200} \sim 0.85 \times 10^{14} M_{\odot}$, fully consistent with the predictions made by the cluster sizerichness relation of Hansen et al. (2009). From this point of view J0454 is indistinguishable from normal clusters, forming either a rich fossil group or a poor fossil cluster. The X-ray halo can be described by a classic $\beta$-model and is only marginally offset $(24 \mathrm{kpc})$ from the brightest group galaxy. However, the velocity dispersion of $316 \mathrm{~km} \mathrm{~s}^{-1}$ is lower than the one measured from weak lensing (476 $\left.\mathrm{km} \mathrm{s}^{-1}\right)$ and spectroscopy $\left(480 \mathrm{~km} \mathrm{~s}^{-1}\right)$, and so is $M_{200}\left(0.34 \times 10^{14} M_{\odot}\right)$. The low X-ray halo temperature of $1.1 \mathrm{keV}$ also favours a smaller structure with $\sim 330 \mathrm{~km} \mathrm{~s}^{-1}$.

Peculiarities arise when analysing the brightest group galaxy with respect to its environment. It not located at the spatial centre of elliptical galaxies and shows a significantly different velocity than the mean velocity of the ellipticals. This indicates a different 
origin of E0454 from the surrounding galaxies. More evidence for this hypothesis is provided by the strongly lensed galaxy near the core of E0454. We constrained its redshift to $z=2.1 \pm 0.3$ and determined an Einstein radius of 2'.37. The weak lensing velocity dispersion of $476 \mathrm{~km} \mathrm{~s}^{-1}$ corresponds to an Einstein radius of $\theta_{\mathrm{E}}=5^{\prime \prime}$.28, meaning that E0454 cannot be located at the centre of the dark matter halo of J0454. Even stronger evidence comes from the external shear required to fit the position of the counter image. About $15 \%$ of the shear can be attributed to the background cluster MS0451, and about 30\% to individual galaxies near E0454, but the dominant contribution must come from J0454 itself. This can only be explained if E0454 is not at the centre of the gravitational potential. If we describe the density profile with NFW, then the projected distance between E0454 and the halo centre must be at least $\sim 120 \mathrm{kpc}$. Whereas such offsets have been shown to be common for other groups and clusters (Oegerle \& Hill 2001; von der Linden et al. 2007; Skibba et al. 2010) this has not yet been reported for fossils.

An explanation that reconciles all observations is that E0454 is currently infalling for the first time into the sparse cluster J0454, seeding the brightest cluster galaxy. An alternative interpretation is that J0454 is of filamentary nature, projected along the line of sight, and galaxies therein stream towards the denser fossil core. Both scenarios explain why the X-ray halo appears associated with E0454, has undisturbed isophotes, no shock fronts, a low temperature and a velocity dispersion and mass that fits a smaller group. This hypothesis is only possible because of the presence and properties of the strong lens, ruling out that E0454 is at the gravitational centre. Without the lens all data would form a consistent picture.

Recently, Lopes de Oliveira et al. (2010) have demonstrated for the fossil UGC 842 that it segregates into two groups with $\sigma_{v} \sim 220 \mathrm{~km} \mathrm{~s}^{-1}$ each, separated by about $820 \mathrm{~km} \mathrm{~s}^{-1}$. Contrary to J0454 with a comparatively low temperature of $1.1 \mathrm{keV}$, UGC 842 shows a, with respect to the velocity dispersion, increased temperature of $1.9 \mathrm{keV}$, which has been interpreted as a sign of an advanced interaction or merging state.

\subsection{Future observations}

Our Subaru/Suprime-Cam images are significantly deeper than those of any other fossil system investigated so far, reaching $10 \sigma$-limits of 24.9 in $z$-band down to 26.6 in $B$-band. Hence this data set could probe the luminosity function $\sim 9$ mag below the BGG, provided deep spectroscopic data are available to remove objects with very similar redshifts. The existing spectra are limited to $I<21.5 \mathrm{mag}$ and complete to only about $40 \%$ at this depth. Hence we limited our analysis to galaxies with $I<22$ mag. With several hours of exposure time at 4-8 m telescopes we could push the spectroscopic limit by $\sim 2.5 \mathrm{mag}$, which would enable us to present an uncontaminated luminosity function extending down to dwarf ellipticals. Numerous of those are seen in the data with colours matching that of the red sequence. With a better spectroscopic sampling we could remove all line of sight contamination and construct a complete red sequence down to much fainter magnitudes, and a fairly complete sample of blue galaxies. There are also possibilities that we could resolve J0454 from a dynamical point of view into members belonging to the fossil component, and into galaxies belonging to the sparse surrounding. If both components have indeed separate origins, then one could attempt to identify stellar populations of different age and composition.

Significantly deeper X-ray data could be used to better determine the offset with respect to the BGG. We could also look for temperature variations and changes in the chemical composition of the gas, which would tell us more about the different origins of the sparse cluster and the infalling group.

Lastly, deeper space-based observations could double the number density of lensed galaxies and we could attempt to obtain direct evidence for the separation of J0454 and E0454 in the mass reconstructions. However, given the aged detectors of the HST/ACS instrument this will be a difficult endeavour.

Acknowledgements. M.S. thanks Bodo Ziegler at ESO and the staff at Paranal for the prompt execution of the DDT programme, and Helen Eckmiller, Bernard Fort, Sarah Hansen, Stefan Hilbert, Satoshi Miyazaki and Achille Nucita for their expertise and helpfulness concerning various aspects of this work. Andrew Cardwell, Karianne Holhjem and Peter Schneider provided very useful comments on the manuscript. We thank the anonymous referee for very helpful suggestions that improved the paper significantly. Author contributions: M.S. did the scientific analysis, obtained the VLT spectrum, reduced the Subaru, VLT and the XMM data, discovered the strong lens system and wrote most parts of the manuscript. S.S. did the strong lens modelling, based upon a code developed by herself and by A.H. T.S. reduced the HST/ACS data and provided the shear catalogue, while H.H. complemented it with photometric redshifts. T.E. provided the stacks of the Elixir pre-processed CFHT $u^{*}$ griz images. Some figures in this paper were made with the plotting tool WIP (Morgan 1995). The authors wish to recognize and acknowledge the very significant cultural role and reverence that the summit of Mauna Kea has always had within the indigenous Hawaiian community. We are most fortunate to have the opportunity to conduct observations from this mountain. M.S. acknowledges support by the German Ministry for Science and Education (BMBF) through DESY under the project 05AV5PDA/3 and the Deutsche Forschungsgemeinschaft (DFG) in the frame of the Schwerpunktprogramm SPP 1177 "Galaxy Evolution". S.S. is supported in part through the DFG under project SCHN 342/7-1. T.S. acknowledges financial support from the Netherlands Organisation for Scientific Research (NWO). H.H. is supported by DUEL-RTN, MRTN-CT-2006-036133, and A.H. by the DFG cluster of excellence "Origin and Structure of the Universe".

\section{References}

Argence, B., \& Lamareille, F. 2009, A\&A, 495, 759

Bertin, E. 2006, in Astronomical Data Analysis Software and Systems XV, ed. C. Gabriel, C. Arviset, D. Ponz, \& S. Enrique, ASP Conf. Ser., 351, 112 Bertin, E., \& Arnouts, S. 1996, A\&AS, 117, 393

Blanton, M. R., \& Roweis, S. 2007, AJ, 133, 734

Böhringer, H., Schuecker, P., Guzzo, L., et al. 2004, A\&A, 425, 367 Boschin, W. 2002, A\&A, 396, 397

Boylan-Kolchin, M., Ma, C.-P., \& Quataert, E. 2008, MNRAS, 383, 93

Burns, J. O., Hallman, E. J., Gantner, B., Motl, P. M., \& Norman, M. L. 2008, ApJ, 675, 1125

Carlberg, R. G., Yee, H. K. C., \& Ellingson, E. 1997, ApJ, 478, 462

Chandrasekhar, S. 1943, ApJ, 97, 255

Cypriano, E. S., Mendes de Oliveira, C. L., \& Sodré, L. J. 2006, AJ, 132, 514

Danese, L., de Zotti, G., \& di Tullio, G. 1980, A\&A, 82, 322

Dariush, A., Khosroshahi, H. G., Ponman, T. J., et al. 2007, MNRAS, 382, 433

Desroches, L., Quataert, E., Ma, C., \& West, A. A. 2007, MNRAS, 377, 402

Dunkley, J., Bucher, M., Ferreira, P. G., Moodley, K., \& Skordis, C. 2005, MNRAS, 356, 925

Edge, A. C. 2001, MNRAS, 328, 762

Elíasdóttir, Á., Limousin, M., Richard, J., et al. 2007 [arXiv:0710. 5636]

Erben, T., Hildebrandt, H., Lerchster, M., et al. 2009, A\&A, 493, 1197

Erben, T., Schirmer, M., Dietrich, J., et al. 2005, AN, 326, 432

Erben, T., van Waerbeke, L., Bertin, E., Mellier, Y., \& Schneider, P. 2001, A\&A, 366,717

Fassnacht, C. D., Kocevski, D. D., Auger, M. W., et al. 2008, ApJ, 681, 1017

Gladders, M., \& Yee, H. 2000, AJ, 120, 2148

Goto, T., Yamauchi, C., Fujita, Y., et al. 2003, MNRAS, 346, 601

Halkola, A., Hildebrandt, H., Schrabback, T., et al. 2008, A\&A, 481, 65

Halkola, A., Seitz, S., \& Pannella, M. 2006, MNRAS, 372, 1425

Hansen, S. M., McKay, T. A., Wechsler, R. H., et al. 2005, ApJ, 633, 122

Hansen, S. M., Sheldon, E. S., Wechsler, R. H., \& Koester, B. P. 2009, ApJ, 699, 1333

Harrison, E. R. 1974, ApJ, 191, L51

Hashimoto, Y., Henry, J. P., \& Boehringer, H. 2008, MNRAS, 390, 1562

Hildebrandt, H., Pielorz, J., Erben, T., et al. 2009, A\&A, 498, 725

Hoekstra, H., Franx, M., Kuijken, K., \& Squires, G. 1998, ApJ, 504, 636

Hogg, D. W., Baldry, I. K., Blanton, M. R., \& Eisenstein, D. J. 2002 [arXiv:astro-ph/0210394]

Holhjem, K., Schirmer, M., \& Dahle, H. 2009, A\&A, 504, 1 
Jeltema, T. E., Mulchaey, J. S., Lubin, L. M., Rosati, P., \& Böhringer, H. 2006, ApJ, 649, 649

Jeltema, T. E., Mulchaey, J. S., Lubin, L. M., \& Fassnacht, C. D. 2007, ApJ, 658, 865

Johnston, D. E., Sheldon, E. S., Wechsler, R. H., et al. 2007 [arXiv: 0709. 1159]

Jones, L. R., Ponman, T. J., Horton, A., et al. 2003, MNRAS, 343, 627

Kaiser, N., Squires, G., \& Broadhurst, T. 1995, ApJ, 449, 460

Kalberla, P. M. W., Burton, W. B., Hartmann, D., et al. 2005, A\&A, 440, 775

Kassiola, A., \& Kovner, I. 1993, ApJ, 417, 450

Kennicutt, Jr., R. C. 1998, ARA\&A, 36, 189

Khosroshahi, H. G., Jones, L. R., \& Ponman, T. J. 2004, MNRAS, 349, 1240

Khosroshahi, H. G., Maughan, B. J., Ponman, T. J., \& Jones, L. R. 2006a, MNRAS, 369, 1211

Khosroshahi, H. G., Ponman, T. J., \& Jones, L. R. 2006b, MNRAS, 372, L68

Khosroshahi, H. G., Ponman, T. J., \& Jones, L. R. 2007, MNRAS, 377, 595

Koch, A., Odenkirchen, M., Grebel, E. K., \& Caldwell, J. A. R. 2004, Astron. Nachr., 325, 299

Koopmans, L. V. E., Treu, T., Bolton, A. S., Burles, S., \& Moustakas, L. A. 2006, ApJ, 649, 599

La Barbera, F., de Carvalho, R. R., de la Rosa, I. G., et al. 2009, AJ, 137, 3942

Limousin, M., Cabanac, R., Gavazzi, R., et al. 2009, A\&A, 502, 445

Lopes de Oliveira, R., Carrasco, E. R., Mendes de Oliveira, C., et al. 2010, AJ, 139,216

Luppino, G. A., \& Kaiser, N. 1997, ApJ, 475, 20

Magnier, G., \& Cuillandre, J.-C. 2004, PASP, 116, 449

Manfroid, J., \& Selman, F. 2001, The Messenger, 104, 16

McKean, J. P., Auger, M. W., Koopmans, L. V. E., et al. 2010, MNRAS, 404, 749

Mendes de Oliveira, C. L., Cypriano, E. S., \& Sodré, L. J. 2006, AJ, 131, 158

Milosavljević, M., Miller, C. J., Furlanetto, S. R., \& Cooray, A. 2006, ApJ, 637, L9

Miyazaki, S., Komiyama, Y., Sekiguchi, M., et al. 2002, PASJ, 54, 833

Momcheva, I., Williams, K., Keeton, C., \& Zabludoff, A. 2006, ApJ, 641, 169

Moran, S. M., Ellis, R. S., Treu, T., et al. 2007, ApJ, 671, 1503

Morgan, J. 1995, ASPC, 77, 129

Morrison, R., \& McCammon, D. 1983, ApJ, 270, 119

Mulchaey, J. S. 2000, ARA\&A, 38, 289

Mulchaey, J. S., \& Zabludoff, A. I. 1998, ApJ, 496, 73

Mulchaey, J. S., Lubin, L. M., Fassnacht, C., Rosati, P., \& Jeltema, T. E. 2006, ApJ, 646, 133

Nath, B. B. 2008, MNRAS, 387, L50

Navarro, J., Frenk, C., \& White, S. 1997, ApJ, 486, 493

Nusser, A., \& Sheth, R. K. 1999, MNRAS, 303, 685
Oegerle, W. R., \& Hill, J. M. 2001, AJ, 122, 2858

Okumura, T., Jing, Y. P., \& Li, C. 2009, ApJ, 694, 214

Parry, O. H., Eke, V. R., \& Frenk, C. S. 2009, MNRAS, 396, 1972

Peng, C. Y., Ho, L. C., Impey, C. D., \& Rix, H. W. 2002, AJ, 124, 266

Pickles, A. J. 1998, PASP, 110, 863

Ponman, T. J., Allan, D. J., Jones, L. R., et al. 1994, Nature, 369, 462

Quilis, V., Moore, B., \& Bower, R. 2000, Science, 288, 1617

Rakić, A., Räsänen, S., \& Schwarz, D. J. 2006, MNRAS, 369, L27

Reiprich, T. H., \& Böhringer, H. 2002, ApJ, 567, 716

Rykoff, E. S., McKay, T. A., Becker, M. R., et al. 2008, ApJ, 675, 1106

Sanderson, A. J. R., Edge, A. C., \& Smith, G. P. 2009, MNRAS, 398, 1698

Santos, W. A., Mendes de Oliveira, C., \& Sodré, L. J. 2007, AJ, 134, 1551

Sarazin, C. L. 1988, X-ray emission from clusters of galaxies (Cambridge: Cambridge University Press), Cambridge Astrophys. Ser.

Scannapieco, C., White, S. D. M., Springel, V., \& Tissera, P. B. 2009, MNRAS, 396, 696

Schirmer, M., Erben, T., Hetterscheidt, M., \& Schneider, P. 2007, A\&A, 462, 875

Schlegel, D. J., Finkbeiner, D. P., \& Davis, M. 1998, ApJ, 500, 525

Schrabback, T., Erben, T., Simon, P., et al. 2007, A\&A, 468, 823

Schrabback, T., Hartlap, J., Joachimi, B., et al. 2009, A\&A, accepted [arXiv:0911.0053]

Scoville, N., Abraham, R. G., Aussel, H., et al. 2007, ApJS, 172, 38

Seitz, S., \& Schneider, P. 2001, A\&A, 374, 740

Sheldon, E. S., Johnston, D. E., Masjedi, M., et al. 2009a, ApJ, 703, 2232

Sheldon, E. S., Johnston, D. E., Scranton, R., et al. 2009b, ApJ, 703, 2217

Skibba, R. A., van den Bosch, F. C., Yang, X., et al. 2010, MNRAS, submitted [arXiv: 1001.4533$]$

Stanek, R., Evrard, A. E., Böhringer, H., Schuecker, P., \& Nord, B. 2006, ApJ, 648,956

Sun, M., Forman, W., Vikhlinin, A., et al. 2004, ApJ, 612, 805

Suyu, S. H., Marshall, P. J., Hobson, M. P., \& Blandford, R. D. 2006, MNRAS, 371,983

Vikhlinin, A., McNamara, B. R., Hornstrup, A., et al. 1999, ApJ, 520, L1

Voevodkin, A., Borozdin, K., Heitmann, K., et al. 2010, ApJ, 708, 1376

Voges, W., Aschenbach, B., Boller, T., et al. 1999, A\&A, 349, 389

von Benda-Beckmann, A. M., D’Onghia, E., Gottlöber, S., et al. 2008, MNRAS, 386, 2345

von der Linden, A., Best, P. N., Kauffmann, G., \& White, S. D. M. 2007, MNRAS, 379, 867

Watson, M. G., Schröder, A. C., Fyfe, D., et al. 2009, A\&A, 493, 339

Yoshioka, T., Furuzawa, A., Takahashi, S., et al. 2004, Adv. Space Res., 34, 2525

Zabludoff, A. I., \& Mulchaey, J. S. 1998, ApJ, 496, 39 
Appendix A:

Table A.1. Members of J0454, ordered by increasing separation $r$ from E0454.

\begin{tabular}{|c|c|c|c|c|c|c|c|c|c|c|c|}
\hline No. & $\alpha(2000.0)$ & $\delta(2000.0)$ & $r\left[^{\prime}\right]$ & $I$ & $B-V$ & $V-I$ & $M_{g}$ & $M_{i}$ & $z$ (phot) & $z$ (spec) & Type \\
\hline 1 & $04: 54: 00.62$ & $\begin{array}{l}-03: 08: 33.8 \\
\end{array}$ & 0.00 & 16.61 & 1.65 & 0.97 & -23.23 & -24.09 & 0.31 & 0.25940 & $\mathrm{E}$ \\
\hline 2 & 04:54:01.11 & $-03: 08: 35.1$ & 0.11 & 20.65 & 1.50 & 0.91 & -19.58 & -20.49 & 0.30 & 0.26449 & S0 \\
\hline 3 & 04:54:00.63 & $-03: 08: 24.8$ & 0.14 & 20.80 & 1.47 & 0.85 & -19.40 & -20.26 & 0.27 & - & E \\
\hline 4 & 04:54:00.56 & $-03: 08: 20.6$ & 0.22 & 19.64 & 1.57 & 0.97 & -21.02 & -21.94 & 0.31 & 0.25962 & E \\
\hline 5 & 04:53:59.74 & $-03: 08: 17.9$ & 0.34 & 19.85 & 1.43 & 0.91 & -20.75 & -21.54 & 0.26 & - & E \\
\hline 6 & 04:54:01.17 & $-03: 09: 04.0$ & 0.51 & 19.89 & 1.52 & 0.99 & -20.58 & -21.25 & 0.31 & - & So \\
\hline 7 & 04:53:59.98 & $-03: 09: 09.6$ & 0.61 & 20.52 & 1.52 & 0.86 & -19.70 & -20.40 & 0.31 & - & E \\
\hline 8 & 04:54:01.19 & $-03: 07: 51.0$ & 0.72 & 21.13 & 1.41 & 0.89 & -18.63 & -19.45 & 0.28 & - & E \\
\hline 9 & $04: 54: 00.26$ & $-03: 07: 47.3$ & 0.78 & 20.44 & 1.49 & 0.98 & -19.30 & -20.24 & 0.29 & - & E \\
\hline $10 *$ & $04: 53: 57.36$ & $-03: 08: 48.7$ & 0.85 & 19.44 & 1.62 & 1.10 & -20.96 & -21.99 & 0.31 & 0.26206 & $\mathrm{Sa}$ \\
\hline 11 & 04:53:58.70 & $-03: 09: 34.5$ & 1.12 & 20.35 & 1.37 & 0.87 & -19.59 & -20.40 & 0.26 & 0.25970 & E \\
\hline 12 & 04:54:04.84 & $-03: 08: 09.6$ & 1.12 & 21.02 & 1.32 & 0.79 & -18.86 & -19.56 & 0.25 & 0.25796 & So \\
\hline $13 *$ & 04:54:03.72 & $-03: 07: 41.4$ & 1.16 & 20.42 & 1.01 & 0.72 & -19.63 & -20.13 & 0.25 & 0.26272 & $\mathrm{Sb}$ \\
\hline 14 & 04:53:57.69 & $-03: 09: 31.2$ & 1.20 & 21.06 & 1.51 & 0.85 & -19.03 & -19.93 & 0.28 & 0.25880 & SBa \\
\hline 15 & 04:53:55.81 & $-03: 08: 21.2$ & 1.22 & 20.34 & 1.44 & 0.91 & -19.62 & -20.52 & 0.30 & 0.26158 & E \\
\hline $16^{*}$ & 04:54:02.70 & $-03: 09: 50.1$ & 1.37 & 21.59 & 0.64 & 0.18 & -19.25 & -19.38 & 0.22 & 0.26341 & Irr \\
\hline $17 *$ & 04:54:04.08 & $-03: 07: 29.7$ & 1.37 & 21.41 & 0.58 & 0.31 & -18.89 & -19.03 & 0.23 & 0.26476 & $\mathrm{Sa}$ \\
\hline $18^{*}$ & 04:54:06.30 & $-03: 08: 38.4$ & 1.41 & 19.40 & 1.60 & 1.07 & -20.70 & -21.73 & 0.34 & 0.25835 & $\mathrm{Sa}$ \\
\hline 19 & $04: 53: 55.20$ & $-03: 07: 52.8$ & 1.52 & 19.79 & 1.56 & 0.96 & -20.10 & -21.09 & 0.31 & 0.26263 & $\mathrm{Sa}$ \\
\hline 20 & 04:53:57.70 & $-03: 07: 09.4$ & 1.58 & 19.09 & 1.58 & 0.97 & -21.03 & -22.02 & 0.31 & 0.26018 & E \\
\hline 21 & 04:53:55.68 & $-03: 07: 21.7$ & 1.72 & 19.75 & 1.57 & 0.92 & -20.39 & -21.35 & 0.29 & - & $\mathrm{SBb}$ \\
\hline $22 *$ & 04:54:05.43 & $-03: 09: 48.3$ & 1.72 & 20.84 & 0.85 & 0.38 & -19.58 & -19.85 & 0.26 & 0.26451 & $\mathrm{Sc}$ \\
\hline $23^{*}$ & 04:54:07.82 & $-03: 08: 24.9$ & 1.79 & 21.63 & 1.14 & 0.64 & -18.53 & -19.08 & 0.32 & 0.26333 & $\mathrm{Sc}$ \\
\hline 24 & 04:54:00.73 & $-03: 10: 22.6$ & 1.81 & 21.32 & 1.43 & 0.91 & -18.55 & -19.38 & 0.27 & - & $\mathrm{Sa}$ \\
\hline 25 & 04:53:56.91 & $-03: 10: 09.2$ & 1.84 & 21.95 & 1.20 & 0.73 & -18.01 & -18.72 & 0.35 & 26568 & $\mathrm{SBc}$ \\
\hline 26 & 04:54:04.36 & $-03: 06: 56.0$ & 1.87 & 21.33 & 1.39 & 0.94 & -18.55 & -19.41 & 0.26 & - & $\mathrm{Sb}$ \\
\hline 27 & 04:54:06.95 & $-03: 07: 28.5$ & 1.91 & 19.56 & 1.51 & 0.95 & -20.98 & -21.90 & 0.30 & 0.26177 & So \\
\hline 28 & 04:53:54.88 & $-03: 07: 09.9$ & 2.00 & 21.66 & 1.31 & 0.81 & -18.43 & -19.17 & 0.34 & - & $\mathrm{Sb}$ \\
\hline 29 & 04:54:08.62 & $-03: 08: 22.3$ & 2.00 & 19.54 & 1.51 & 0.92 & -20.47 & -21.40 & 0.30 & 26179 & So \\
\hline 30 & 04:53:55.39 & $-03: 10: 11.5$ & 2.09 & 21.70 & 1.37 & 0.81 & -18.14 & -18.94 & 0.25 & - & So \\
\hline 31 & 04:53:55.36 & $-03: 06: 54.4$ & 2.11 & 21.21 & 1.37 & 0.79 & -18.83 & -19.56 & 0.29 & - & $\mathrm{Sa}$ \\
\hline 32 & 04:53:59.49 & $-03: 10: 43.8$ & 2.18 & 20.25 & 1.37 & 0.89 & -20.05 & -20.87 & 0.29 & - & $\mathrm{SBb}$ \\
\hline 33 & 04:54:00.64 & $-03: 10: 45.9$ & 2.20 & 19.34 & 1.51 & 0.97 & -20.69 & -21.65 & 0.30 & - & So \\
\hline 34 & $04: 54: 02.35$ & $-03: 10: 44.2$ & 2.21 & 18.56 & 1.59 & 1.02 & -21.71 & -22.69 & 0.33 & - & E \\
\hline 35 & 04:54:00.43 & $-03: 06: 20.2$ & 2.22 & 20.19 & 1.52 & 0.98 & -19.76 & -20.71 & 0.31 & - & $\mathrm{Sa}$ \\
\hline 36 & $04: 53: 55.25$ & $-03: 06: 37.8$ & 2.35 & 19.40 & 1.58 & 0.94 & -20.70 & -21.65 & 0.32 & - & E \\
\hline 37 & 04:54:05.76 & $-03: 10: 52.1$ & 2.63 & 21.83 & 1.32 & 0.85 & -17.95 & -18.72 & 0.22 & - & $\mathrm{Sa}$ \\
\hline 38 & 04:54:01.61 & $-03: 05: 51.9$ & 2.70 & 20.92 & 1.39 & 0.90 & -18.98 & -19.80 & 0.31 & .25915 & E \\
\hline 39 & 04:54:06.31 & $-03: 06: 05.0$ & 2.85 & 21.57 & 1.23 & 0.87 & -18.29 & -19.03 & 0.26 & - & $\mathrm{Sb}$ \\
\hline $40 *$ & $04: 54: 10.03$ & $-03: 10: 14.6$ & 2.88 & 19.54 & 1.09 & 0.91 & -20.59 & -21.26 & 0.31 & 0.26021 & $\mathrm{Sa}$ \\
\hline 41 & 04:54:03.81 & $-03: 11: 30.1$ & 3.04 & 21.48 & 1.42 & 0.84 & -18.56 & -19.36 & 0.25 & - & $\mathrm{SBc}$ \\
\hline 42 & 04:53:56.61 & $-03: 05: 39.9$ & 3.06 & 19.45 & 1.51 & 0.93 & -20.74 & -21.62 & 0.29 & - & E \\
\hline 43 & $04: 53: 48.29$ & $-03: 09: 08.7$ & 3.13 & 19.98 & 1.44 & 0.88 & -20.61 & -21.46 & 0.36 & 0.26410 & $\mathrm{SBb}$ \\
\hline $44 *$ & 04:54:13.09 & $-03: 09: 34.7$ & 3.26 & 20.78 & 1.04 & 0.74 & -19.51 & -20.15 & 0.32 & 0.26060 & Irr \\
\hline 45 & 04:54:14.09 & $-03: 07: 58.3$ & 3.40 & 18.46 & 1.60 & 0.91 & -22.06 & -23.05 & 0.30 & - & So \\
\hline 46 & 04:53:46.78 & $-03: 09: 24.5$ & 3.56 & 19.21 & 1.48 & 0.86 & -21.99 & -22.78 & 0.27 & - & So \\
\hline 47 & $04: 54: 14.76$ & $-03: 07: 42.2$ & 3.62 & 21.04 & 1.34 & 0.81 & -18.99 & -19.69 & 0.25 & - & $\mathrm{Sa}$ \\
\hline 48 & $04: 53: 58.25$ & $-03: 12: 31.1$ & 4.00 & 21.01 & 1.29 & 0.81 & -19.15 & -19.95 & 0.32 & - & $\mathrm{Sb}$ \\
\hline $49 *$ & $04: 53: 46.78$ & $-03: 06: 12.7$ & 4.18 & 20.87 & 0.83 & 0.40 & -19.72 & -19.96 & 0.23 & 0.26221 & Irr \\
\hline 50 & 04:54:09.42 & $-03: 04: 56.6$ & 4.23 & 21.87 & 1.29 & 0.78 & -18.08 & -18.71 & 0.24 & - & So \\
\hline $51^{*}$ & $04: 53: 45.16$ & $-03: 06: 43.0$ & 4.28 & 21.08 & 1.00 & 0.60 & -19.84 & -20.21 & 0.27 & 0.25720 & Irr \\
\hline 52 & $04: 53: 47.63$ & $-03: 05: 21.3$ & 4.56 & 19.68 & 1.44 & 0.89 & -20.38 & -21.25 & 0.28 & - & $\mathrm{Sa}$ \\
\hline $53 *$ & 04:54:11.76 & $-03: 04: 51.7$ & 4.62 & 20.79 & 1.32 & 0.72 & -19.15 & -19.86 & 0.22 & 0.26083 & So \\
\hline $54 *$ & 04:54:06.07 & $-03: 04: 05.0$ & 4.68 & 21.03 & 0.89 & 0.41 & -19.80 & -20.04 & 0.24 & 0.26156 & $\mathrm{Sc}$ \\
\hline 55 & $04: 53: 41.86$ & $-03: 08: 38.1$ & 4.69 & 19.78 & 1.28 & 0.92 & -20.48 & -21.24 & 0.30 & - & SBa \\
\hline $56^{*}$ & $04: 53: 41.47$ & $-03: 08: 11.8$ & 4.80 & 20.85 & 1.07 & 0.60 & -19.50 & -19.97 & 0.26 & 0.26178 & $\mathrm{SBb}$ \\
\hline $57 *$ & $04: 54: 21.44$ & $-03: 08: 29.0$ & 5.19 & 20.61 & 0.94 & 0.33 & -19.75 & -20.05 & 0.22 & 0.25968 & $\mathrm{Sb}$ \\
\hline $58^{*}$ & $04: 53: 43.25$ & $-03: 05: 37.3$ & 5.24 & 22.41 & 1.19 & 0.97 & -17.20 & -18.14 & 0.49 & 0.25743 & E \\
\hline 59 & $04: 53: 43.11$ & $-03: 05: 28.5$ & 5.35 & 19.45 & 1.47 & 0.89 & -20.82 & -21.68 & 0.28 & - & E \\
\hline 60 & $04: 53: 57.27$ & $-03: 03: 07.8$ & 5.49 & 21.60 & 1.37 & 0.83 & -18.20 & -18.97 & 0.31 & - & $\mathrm{Sa}$ \\
\hline 61 & $04: 53: 56.15$ & $-03: 14: 06.3$ & 5.65 & 19.69 & 1.46 & 0.93 & -20.45 & -21.41 & 0.32 & - & So \\
\hline 62 & 04:53:58.31 & $-03: 14: 14.6$ & 5.71 & 19.58 & 1.44 & 0.88 & -20.69 & -21.54 & 0.29 & - & E \\
\hline $63^{*}$ & $04: 53: 53.27$ & $-03: 03: 02.5$ & 5.81 & 23.37 & 0.60 & 0.30 & -16.84 & -16.97 & 0.26 & 0.25787 & Irr \\
\hline 64 & $04: 53: 42.20$ & $-03: 12: 14.7$ & 5.89 & 19.17 & 1.58 & 0.92 & -21.16 & -22.12 & 0.30 & - & SBa \\
\hline
\end{tabular}

Notes. An asterisk $\left({ }^{*}\right)$ behind the object number indicates that it was not photometrically selected, but added based on its spectroscopic redshift. $M_{g}$ and $M_{i}$ are the absolute magnitudes after extinction- and $k$-correction. The morphological classification is based on the HST/ACS data and was done visually. 


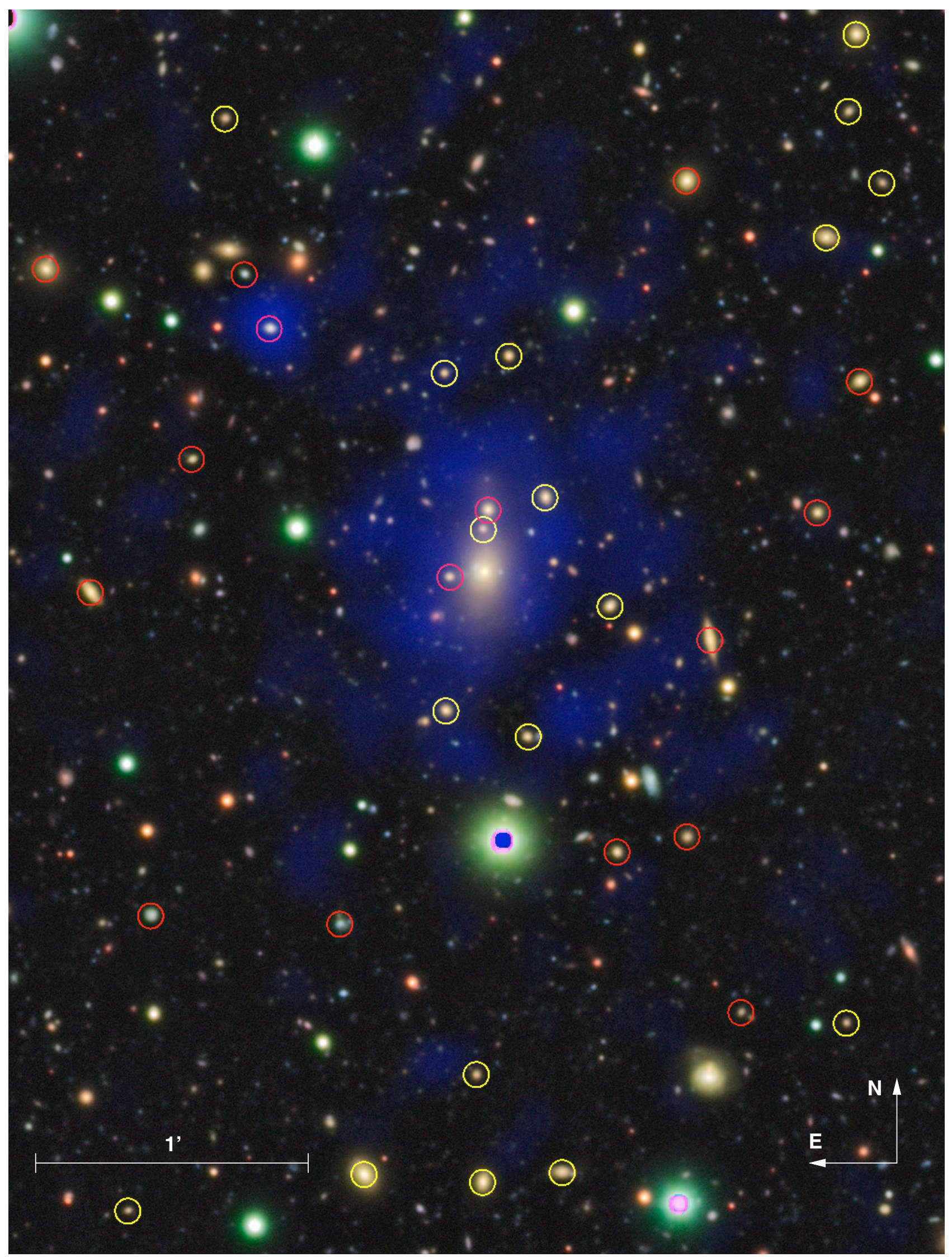

Fig. A.1. Logarithmically scaled Subaru/Suprime-Cam BVR image of the central part of J0454. Galaxies with red circles were spectroscopically confirmed, and those with yellow circles were photometrically selected. The brightest cluster galaxy (E0454) at the centre is left unmarked to show the lensed system. Overlaid in blue is the (smoothed) X-ray emission, diffuse in nature apart from the bright point source to the upper left. 